Supporting Information

\title{
Synthesis of Monodisperse Spiro-Bridged Ladder-Type Oligo-p-Phenylenes
}

Yonggang Wu, Jianyuan Zhang, Zhishan Bo*

Beijing National Laboratory for Molecular Sciences (BNLMS), State Key Laboratory of Polymer Physics and Chemistry, Joint Laboratory of Polymer Science and Materials, Institute of Chemistry, Chinese Academy of Sciences, Beijing, 100080, China

\section{Experimental Section}

General. All chemicals were purchased from commercial suppliers and used without further purification. Compounds $\mathbf{5}^{1}$ and 2,5-dibromobenzene-1,4-dialdehyde ${ }^{2}$ were prepared according to literature procedures. The catalyst precursor $\mathrm{Pd}\left(\mathrm{PPh}_{3}\right)_{4}$ was prepared according to the literature ${ }^{3}$ and stored in a Schlenk tube under nitrogen. All solvents were purified and dried by standard methods. All reactions were monitored by TLC with silica gel 60 F254 (Merck, $0.2 \mathrm{~mm}$ ). Column chromatography was carried out on silica gel (200-300 mesh). ${ }^{1} \mathrm{H}$ and ${ }^{13} \mathrm{C}$ NMR spectra were recorded on a Bruker AV400 or Bruker AV300 spectrometer in $\mathrm{CDCl}_{3}$. The electrochemical behavior of the ladder-type oligomers was investigated by using cyclic voltammetry (CHI 630A Electrochemical Analyzer) with a standard three-electrode electrochemical cell in a $0.1 \mathrm{M}$ tetrabutylammonium tetrafluoroborate solution in $\mathrm{CH}_{3} \mathrm{CN}$ at room temperature under nitrogen atmosphere with a scanning rate of $30 \mathrm{mV} \cdot \mathrm{s}^{-1}$. A glassy carbon working electrode, a Pt wire counter electrode, and an $\mathrm{Ag} / \mathrm{AgNO}_{3}\left(0.01 \mathrm{M}\right.$ in $\left.\mathrm{CH}_{3} \mathrm{CN}\right)$ reference electrode were used. The experiments were calibrated with the standard ferrocene/ferrocenium $(\mathrm{Fc})$ redox system and assumption that the energy level of $\mathrm{Fc}$ is $4.8 \mathrm{eV}$ below vacuum. Electronic absorption spectra were obtained on a SHIMADZU UV-visible spectrometer model UV-1601PC. Fluorescence spectra were recorded on a Varian FLR025. Elemental analyses were performed on a Flash EA 1112 analyzer. TGA (Pyris 1 TGA) and DSC (TA 2910) measurements were carried out under a nitrogen atmosphere at a heating rate of $10{ }^{\circ} \mathrm{C} / \mathrm{min}$ to recorded the thermal gravimetric analysis (TGA) and differential scanning calorimetry (DSC), respectively. The matrix assisted laser desorption ionization time of flight (MALDI-TOF) MS spectrometric measurements were performed on a Bruker BIFLEXIII mass spectrometer.

\section{Synthesis and characterization of Compound 7.}

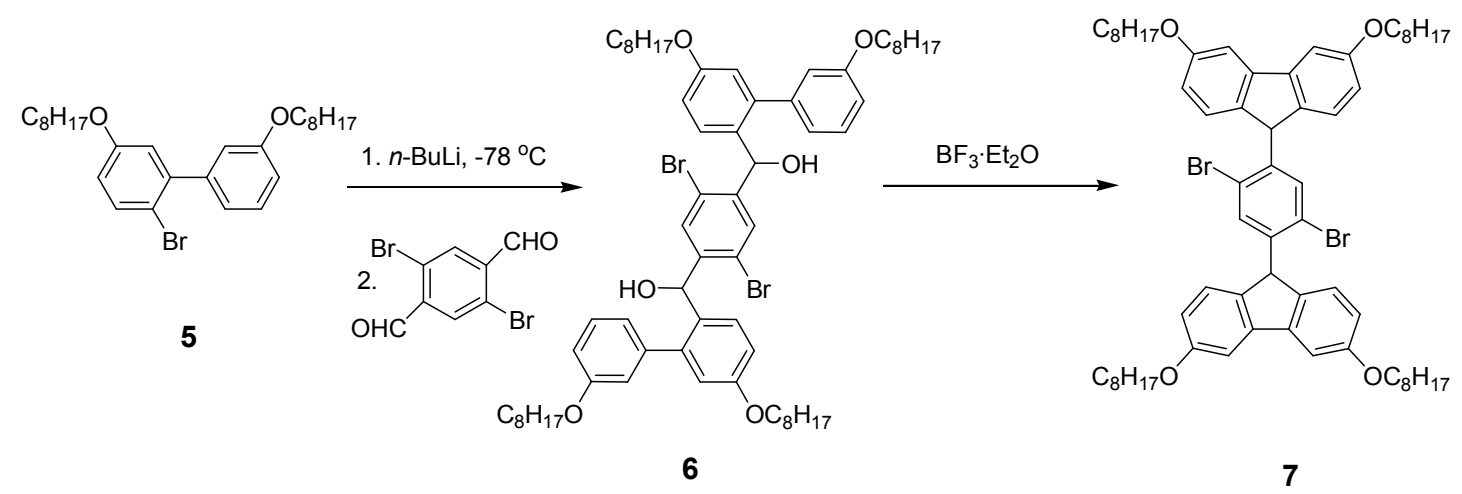

To a solution of $5(3.86 \mathrm{~g}, 7.9 \mathrm{mmol})$ in diethyl ether $(100 \mathrm{~mL})$ was added $n$-BuLi $(3.2 \mathrm{~mL}, 8.0$ mmol) at $-78{ }^{\circ} \mathrm{C}$ under nitrogen. The reaction was keep at $-78{ }^{\circ} \mathrm{C}$ for $15 \mathrm{~min}$, gradually warmed to 0 
${ }^{\circ} \mathrm{C}$. After cooled to $-78^{\circ} \mathrm{C}$ again, a solution of 2,5-dibromoterephthalic aldehyde $(0.96 \mathrm{~g}, 3.3 \mathrm{mmol})$ in THF $(30 \mathrm{~mL})$ was added dropwise. After addition, the mixture was allowed to warm to room temperature and stirred for another two hours. Water $(100 \mathrm{~mL})$ was added to quench the reaction, the organic layer was separated, the aqueous layer was extracted with ether $(50 \mathrm{~mL} \times 2)$, and the combined organic layers were dried over $\mathrm{Na}_{2} \mathrm{SO}_{4}$ and evaporated to dryness. The residue was chromatographically purified on silica gel eluting with $\mathrm{CH}_{2} \mathrm{Cl}_{2}$ /hexane (1:1) increasing to $\mathrm{CH}_{2} \mathrm{Cl}_{2}$ /ether (1:2, v/v) to afford 6 as a yellow solid (1.78 g, 49\%).

To a solution of $6(0.98 \mathrm{~g})$ in $\mathrm{CH}_{2} \mathrm{Cl}_{2}(200 \mathrm{~mL})$ was added $\mathrm{BF}_{3} * \mathrm{Et}_{2} \mathrm{O}(5.0 \mathrm{~mL})$. After the mixture was stirred at room temperature for $20 \mathrm{~min}$, ethanol $(20 \mathrm{~mL})$ was added to destroy the catalyst. The organic layer was then washed with water $(200 \mathrm{~mL} \times 3)$, dried over $\mathrm{Na}_{2} \mathrm{SO}_{4}$, and evaporated to dryness. The residue was chromatographically purified on silica gel eluting with $\mathrm{CH}_{2} \mathrm{Cl}_{2}$ /hexane $(1: 4, \mathrm{v} / \mathrm{v})$ to afford 7 as a colorless solid $(0.79 \mathrm{~g}, 83 \%){ }^{1} \mathrm{H} \mathrm{NMR}\left(400 \mathrm{MHz}, \mathrm{CDCl}_{3}\right): \delta 7.26(\mathrm{~d}, 4 \mathrm{H})$, $7.23(\mathrm{~s}, 4 \mathrm{H}), 6.82(\mathrm{~d}, 4 \mathrm{H}), 6.74(\mathrm{~s}, 2 \mathrm{H}), 5.45(\mathrm{~s}, 2 \mathrm{H}), 4.05(\mathrm{t}, 8 \mathrm{H}), 1.84(\mathrm{~m}, 8 \mathrm{H}), 1.53-1.31$ (broad, $40 \mathrm{H}), 0.90(\mathrm{t}, 12 \mathrm{H}) ;{ }^{13} \mathrm{C} \mathrm{NMR}\left(100 \mathrm{MHz}, \mathrm{CDCl}_{3}\right): \delta 160.0,143.1,143.0,133.1,126.5,115.0,106.3$, 69.0, 51.7, 32.5, 30.1, 29.9, 26.8, 23.3, 14.8. Anal. Calcd. for $\mathrm{C}_{64} \mathrm{H}_{84} \mathrm{Br}_{2} \mathrm{O}_{4}:$ C, 71.36; H, 7.86. Found: C, 71.25; H, 7.89 .

\section{Synthesis and characterization of Compound 8.}
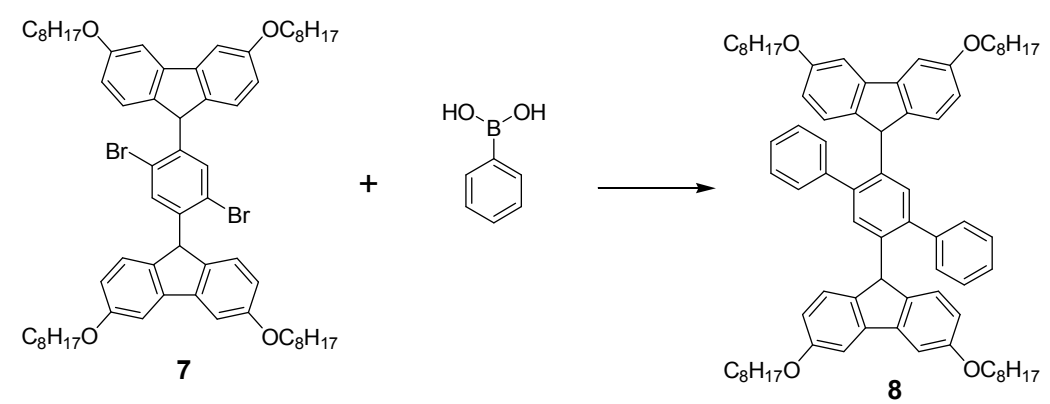

A mixture of 7 ( $0.34 \mathrm{~g}, 0.32 \mathrm{mmol})$, phenylboronic acid $(80 \mathrm{mg}, 0.66 \mathrm{mml}), \mathrm{NaHCO}_{3}(0.48 \mathrm{~g}, 5.7$ $\mathrm{mmol}), \mathrm{H}_{2} \mathrm{O}(6 \mathrm{~mL})$, and THF $(20 \mathrm{~mL})$ was carefully degassed before and after $\left.\mathrm{Pd}_{(\mathrm{PPh}}\right)_{4}(10 \mathrm{mg}$, $0.082 \mathrm{mmol}$ ) was added. The mixture was refluxed for $1 \mathrm{~d}$ under stirring. Water $(50 \mathrm{~mL})$ and $\mathrm{CH}_{2} \mathrm{Cl}_{2}(50 \mathrm{~mL})$ were added, the organic layer was separated, the aqueous layer was extracted with $\mathrm{CH}_{2} \mathrm{Cl}_{2}(50 \mathrm{~mL} \times 2)$, and the combined organic layers were dried over $\mathrm{Na}_{2} \mathrm{SO}_{4}$ and evaporated to dryness. The residue was chromatographically purified on silica gel eluting with $\mathrm{CH}_{2} \mathrm{Cl}_{2}$ /hexane $(1: 2, \mathrm{v} / \mathrm{v})$ to afford 8 as a colorless solid $(0.34 \mathrm{~g}, 97.1 \%) .{ }^{1} \mathrm{H}$ NMR $\left(400 \mathrm{MHz}, \mathrm{CDCl}_{3}\right): \delta 7.38(\mathrm{~d}$, $4 \mathrm{H}), 7.33(\mathrm{t}, 4 \mathrm{H}), 7.25(\mathrm{t}, 2 \mathrm{H}), 7.21(\mathrm{~s}, 4 \mathrm{H}), 7.14(\mathrm{~d}, 4 \mathrm{H}) 6.81(\mathrm{~d}, 4 \mathrm{H}) 6.48(\mathrm{~s}, 2 \mathrm{H}) 5.21(\mathrm{~s}, 2 \mathrm{H}), 4.04$ $(\mathrm{t}, 8 \mathrm{H}), 1.83(\mathrm{~m}, 8 \mathrm{H}), 1.49(\mathrm{~m}, 8 \mathrm{H}) 1.36-1.32$ (broad, 32H), $0.91(\mathrm{t}, 12 \mathrm{H}) ;{ }^{13} \mathrm{C}$ NMR $(100 \mathrm{MHz}$, $\left.\mathrm{CDCl}_{3}\right): \delta 159.5,143.1,143.0,142.0,138.2,130.4,129.8,128.8,127.5,126.4,114.9,105.9,69.0$, 49.0, 32.5, 30.1, 29.9, 26.8, 26.7, 23.3, 14.8. MALDI-TOF, m/z: calcd, 1071.5; found, $1071.3\left(\mathrm{M}^{+}\right)$

\section{Synthesis and characterization of spiro-bridged ladder-type oligomer 1.}




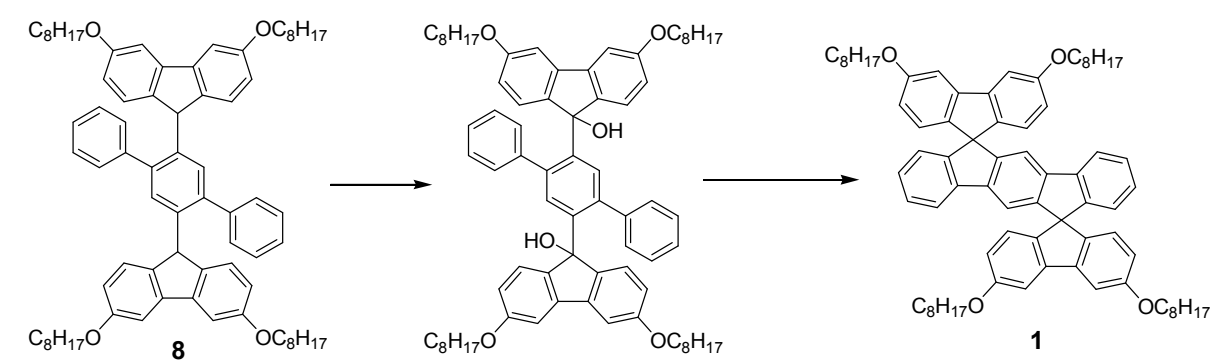

A mixture of $8(0.14 \mathrm{~g}, 0.13 \mathrm{mmol})$, DMSO $(32 \mathrm{~mL}), t$-Butanol $(8 \mathrm{~mL})$, and $\mathrm{KOH}(3.5 \mathrm{~g})$ was stirred vigorously for $12 \mathrm{~h}$ under oxygen atmosphere. Water $(50 \mathrm{~mL})$, acetic acid $(10 \mathrm{~mL})$, and $\mathrm{CH}_{2} \mathrm{Cl}_{2}(50 \mathrm{~mL})$ were added, the organic layer was separated, the aqueous layer was extracted with $\mathrm{CH}_{2} \mathrm{Cl}_{2}(50 \mathrm{~mL} \times 2)$, and the combined organic layers were washed with water $(200 \mathrm{~mL} \times 3)$, dried over $\mathrm{Na}_{2} \mathrm{SO}_{4}$ and evaporated to dryness under reduced pressure. The residue was dissolved in dried $\mathrm{CH}_{2} \mathrm{Cl}_{2}(40 \mathrm{~mL})$, and $\mathrm{BF}_{3} \cdot \mathrm{Et}_{2} \mathrm{O}(0.5 \mathrm{~mL})$ was added dropwise to this mixture. After stirring for 10 min, $10 \mathrm{~mL}$ of ethanol was added, the organic layer was washed with water $(20 \mathrm{~mL} \times 3)$. After removal of the solvent, the residue was chromatographically purified on silica gel eluting with $\mathrm{CH}_{2} \mathrm{Cl}_{2} /$ hexane $(1: 2, \mathrm{v} / \mathrm{v})$ to afford $1(0.13 \mathrm{~g}, 93 \%)$ as a colorless solid. ${ }^{1} \mathrm{H}$ NMR $(400 \mathrm{MHz}$, $\left.\mathrm{CDCl}_{3}\right): \delta 7.49(\mathrm{~d}, 2 \mathrm{H}), 7.37(\mathrm{~s}, 4 \mathrm{H}), 7.18(\mathrm{t}, 2 \mathrm{H}), 7.12(\mathrm{~s}, 2 \mathrm{H}), 6.70(\mathrm{t}, 2 \mathrm{H}), 6.70-6.65(\mathrm{~m}, 10 \mathrm{H})$, $4.05(\mathrm{t}, 8 \mathrm{H}), 1.84(\mathrm{~m}, 8 \mathrm{H}), 1.51(\mathrm{~m}, 8 \mathrm{H}), 1.36-1.31(\mathrm{broad}, 32 \mathrm{H}), 0.90(\mathrm{t}, 12 \mathrm{H}) ;{ }^{13} \mathrm{C}$ NMR $(100$ $\left.\mathrm{MHz}, \mathrm{CDCl}_{3}\right): \delta 159.4,149.5,149.0,143.0,141.6,141.6,141.3,127.4,127.3,124.7,123.6,119.9$, 115.4, 114.4, 105.7, 68.3, 64.5, 31.6, 29.4, 29.3, 26.1, 22.7, 14.1. MALDI-TOF, m/z: calcd, 1067.5; found, $1067.2\left(\mathrm{M}^{+}\right)$.

\section{Synthesis and characterization of Compound 11.}
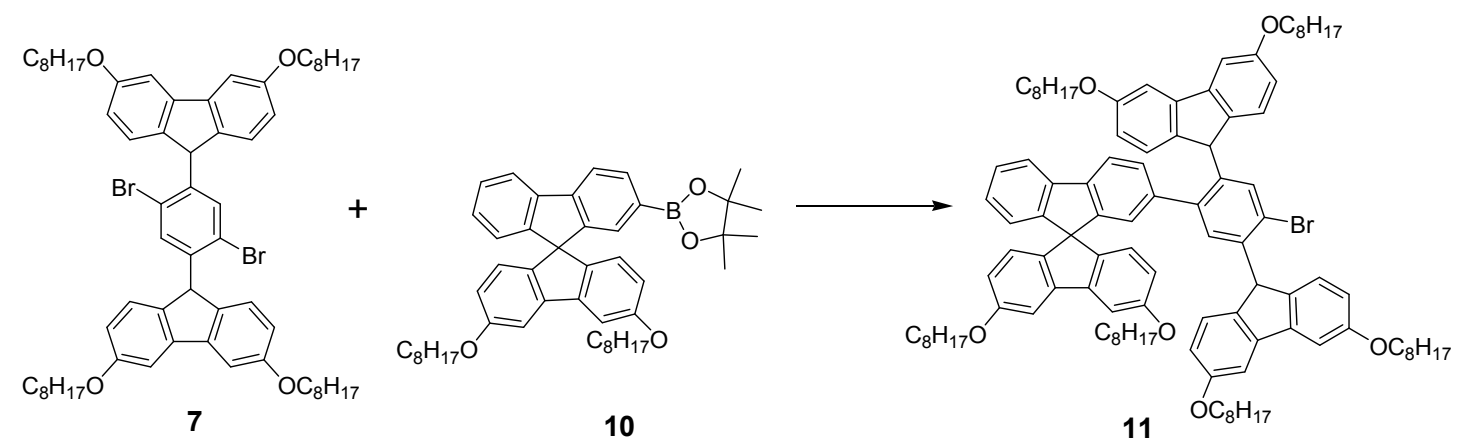

A mixture of 7 (0.90 g, $0.84 \mathrm{mmol}$ ), 3',6'-bis(octyloxy)-9-9'-spirobisfluorenyl-2-boronic acid pinacol ester $10(0.24 \mathrm{~g}, 0.34 \mathrm{mmol}), \mathrm{NaHCO}_{3}(1.0 \mathrm{~g}, 11.9 \mathrm{mmol}), \mathrm{H}_{2} \mathrm{O}(10 \mathrm{~mL})$, and THF $(30 \mathrm{~mL})$ was carefully degassed before and after $\mathrm{Pd}\left(\mathrm{PPh}_{3}\right)_{4}(10 \mathrm{mg})$ was added. The mixture was heated to reflux and stirred under $\mathrm{N}_{2}$ for 24 hours. Water $(50 \mathrm{~mL})$ and $\mathrm{CH}_{2} \mathrm{Cl}_{2}(50 \mathrm{~mL})$ were added, the organic layer was separated, the aqueous layer was extracted with $\mathrm{CH}_{2} \mathrm{Cl}_{2}(50 \mathrm{~mL} \times 2)$, and the combined organic layers were dried over $\mathrm{Na}_{2} \mathrm{SO}_{4}$ and evaporated to dryness. The residue was chromatographically purified on silica gel eluting with $\mathrm{CH}_{2} \mathrm{Cl}_{2}$ /hexane $(1: 3, \mathrm{v} / \mathrm{v})$ to afford $\mathbf{1 1}$ as a colorless solid (0.24 g, 43\%) ${ }^{1} \mathrm{H}$ NMR (400 MHz, $\left.\mathrm{CDCl}_{3}\right): \delta 7.75-7.72(\mathrm{~m}, 2 \mathrm{H}), 7.30-7.12(\mathrm{~m}, 9 \mathrm{H})$ $7.06(\mathrm{t}, 1 \mathrm{H}), 6.76-6.64(\mathrm{~m}, 9 \mathrm{H}), 6.58(\mathrm{~s}, 1 \mathrm{H}), 6.43$ (broad, 5H) $5.45(\mathrm{~s}, 1 \mathrm{H}), 4.63(\mathrm{~s}, 1 \mathrm{H}) 4.01$ (broad, $8 \mathrm{H}$ ), 3.92 (broad, 4H), 1.83 (m, 12H), 1.48 (broad, 12H) 1.35-1.26 (broad, 48H), 0.9 (broad, 18H); ${ }^{13} \mathrm{C}$ NMR (100 MHz, $\left.\mathrm{CDCl}_{3}\right): \delta 159.3,159.0,158.8,149.2,142.8,142.4,142.3142 .0,141.4 .141 .1$. 
$140.9,140.4,139.9,139.4,131.7,128.9,127.8,127.5,125.9,125.5,125.4,124.4,124.0,119.9$, 119.7, 114.1, 105.7, 105.3, 105.2, 68.2.64.6, 51.3, 47.9, 31.8, 29.4, 29.3, 26.1, 22.7, 14.1. Anal. Calcd. for $\mathrm{C}_{105} \mathrm{H}_{131} \mathrm{BrO}_{6}$ : C, 80.37; H, 8.42. Found: $\mathrm{C}, 79.57 ; \mathrm{H}, 8.27$

\section{Synthesis and characterization of Compound 12.}

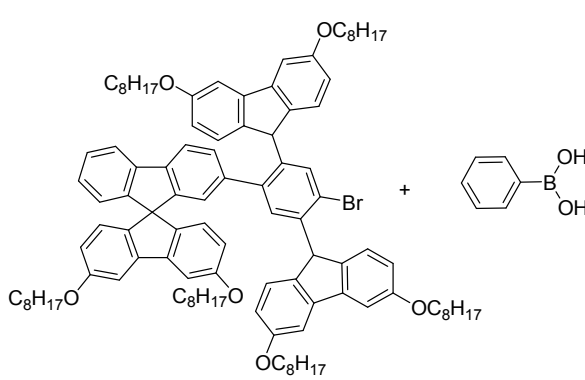

11

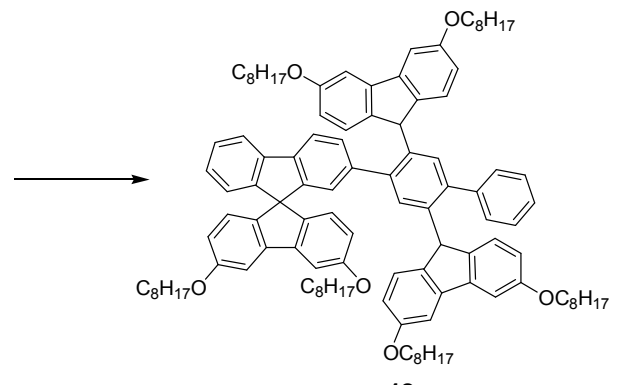

12

A mixture of 11 (74 mg, $0.069 \mathrm{mmol})$, phenylboronic acid (22 mg, $0.18 \mathrm{mmol}), \mathrm{NaHCO}_{3}(0.68 \mathrm{~g}$,

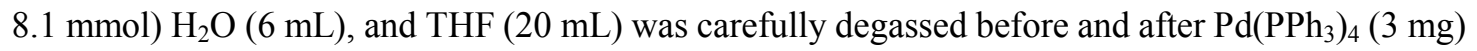
was added. The mixture was heated to reflux and stirred under $\mathrm{N}_{2}$ for 24 hours. Water $(40 \mathrm{~mL})$ and $\mathrm{CH}_{2} \mathrm{Cl}_{2}(40 \mathrm{~mL})$ were added, the organic layer was separated, the aqueous layer was extracted with $\mathrm{CH}_{2} \mathrm{Cl}_{2}(50 \mathrm{~mL} \times 2)$, and the combined organic layers were dried over $\mathrm{Na}_{2} \mathrm{SO}_{4}$ and evaporated to dryness. The residue was chromatographically purified on silica gel eluting with $\mathrm{CH}_{2} \mathrm{Cl}_{2}$ /hexane $(1: 2, \mathrm{v} / \mathrm{v})$ to afford 12 as a colorless solid $(68 \mathrm{mg}, 92 \%){ }^{1} \mathrm{H}$ NMR $\left(400 \mathrm{MHz}, \mathrm{CDCl}_{3}\right): \delta 7.75-7.71$ (m, 2H), 7.31-7.02 (m, 16H), 6.79-6.61 (m, 7H), $6.44(\mathrm{~d}, 2 \mathrm{H}), 6.64-6.29(\mathrm{~m}, 4 \mathrm{H}) 6.29(\mathrm{~s}, 1 \mathrm{H}), 5.12$ (s, 1H) 4.70 (s, 1H) 3.99 (t, 8H), 3.91 (broad, 4H) 1.80 (broad, 12H), 1.48-1.30 (broad, 60H), 0.90 (t, 18H); ${ }^{13} \mathrm{C}$ NMR $\left(100 \mathrm{MHz}, \mathrm{CDCl}_{3}\right): \delta 159.2,158.7,158.6,149.4,149.2,142.8,142.4,142.1$, 142.0, 141.4, 141.3, 141.0, 140.7, 129.6, 128.7, 128.1, 127.6, 127.5, 126.7, 125.6, 124.5, 124.0, 119.8, 119.6, 114.1, 114.0, 105.7, 105.2, 104.9, 68.2, 68.2, 48.3, 48.2 31.8, 29.5, 29.4, 29.3, 29.3, 26.1, 22.7, 14.1. MALDI-TOF, m/z: calcd, 1566.2; found, $1565.8\left(\mathrm{M}^{+}\right)$.

\section{Synthesis and characterization of spiro-bridged ladder-type oligomer 2.}

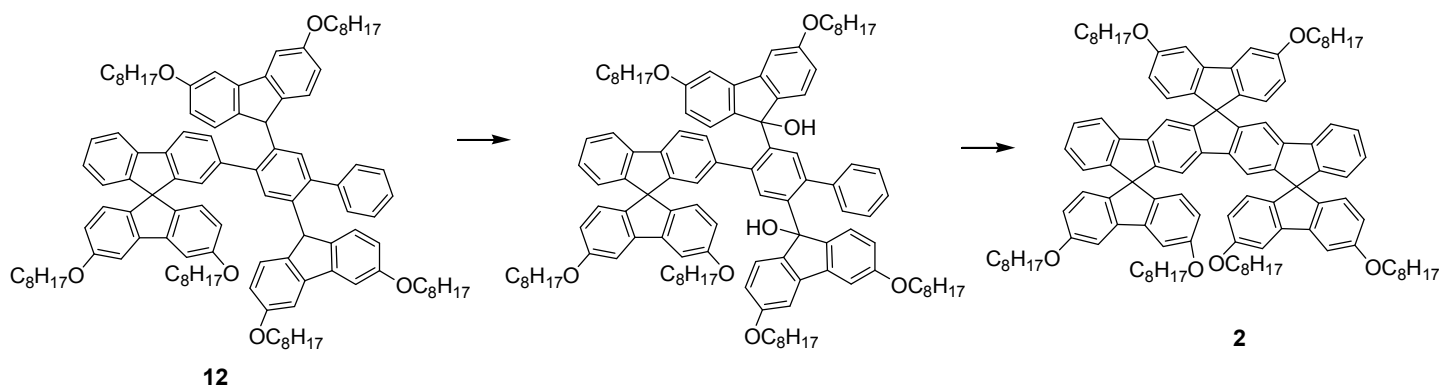

A mixture of $12(68 \mathrm{mg}, 0.043 \mathrm{mmol})$, DMSO (32 mL), $t$-Butanol $(8 \mathrm{~mL})$, and $\mathrm{KOH}(3.5 \mathrm{~g})$ was stirred vigorously for $12 \mathrm{~h}$ under oxygen atmosphere. Water $(50 \mathrm{~mL})$, acetic acid $(10 \mathrm{~mL})$, and $\mathrm{CH}_{2} \mathrm{Cl}_{2}(50 \mathrm{~mL})$ were added, the organic layer was separated, the aqueous layer was extracted with $\mathrm{CH}_{2} \mathrm{Cl}_{2}(50 \mathrm{~mL} \times 2)$, and the combined organic layers were washed with water $(200 \mathrm{~mL} \times 3)$, dried over $\mathrm{Na}_{2} \mathrm{SO}_{4}$ and evaporated to dryness under reduced pressure. The residue was dissolved in dried $\mathrm{CH}_{2} \mathrm{Cl}_{2}(40 \mathrm{~mL})$, and $\mathrm{BF}_{3} \cdot \mathrm{Et}_{2} \mathrm{O}(0.5 \mathrm{~mL})$ was added dropwise to this mixture. After stirring for 10 
min, $10 \mathrm{~mL}$ of ethanol was added, the organic layer was washed with water $(20 \mathrm{~mL} \times 3)$. After removal of the solvent, the residue was chromatographically purified on silica gel eluting with $\mathrm{CH}_{2} \mathrm{Cl}_{2} /$ hexane $(1: 2, \mathrm{v} / \mathrm{v})$ to afford $2(62 \mathrm{mg}, 91 \%)$ as a colorless solid. ${ }^{1} \mathrm{H}$ NMR $(300 \mathrm{MHz}$, $\left.\mathrm{CDCl}_{3}\right): \delta$ 7.44-7.43 (m, 4H), $7.29(\mathrm{~s}, 4 \mathrm{H}), 7.16(\mathrm{t}, 2 \mathrm{H}), 7.07(\mathrm{~s}, 2 \mathrm{H}), 6.96(\mathrm{t}, 2 \mathrm{H}), 6.79(\mathrm{~s}, 2 \mathrm{H}), 6.74$ (s, 4H), 6.64-6.55 (m, 14H), $4.09(\mathrm{t}, 4 \mathrm{H}), 4.00(\mathrm{t}, 8 \mathrm{H}), 1.80(\mathrm{~m}, 12 \mathrm{H}), 1.53-1.31$ (broad, 60H), 0.91 $(\mathrm{t}, 18 \mathrm{H}) ;{ }^{13} \mathrm{C}$ NMR $\left(75 \mathrm{MHz}, \mathrm{CDCl}_{3}\right): \delta 160.1,160.0,150.2,150.0,149.6,143.7,143.6,142.6$, $142.2,142.0,141.9,128.0,125.7,125.3,124.3,120.5,115.9,115.9,115.1,115.0,106.5,106.4$, $69.0,68.9,65.1,32.5,30.4,30.3,30.1,30.0,29.9,26.8,26.8,23.5,23.4,14.8$. MALDI-TOF, m/z: calcd, 1562.2; found, $1562.3\left(\mathrm{M}^{+}\right)$.

\section{Synthesis and characterization of Compound 13.}

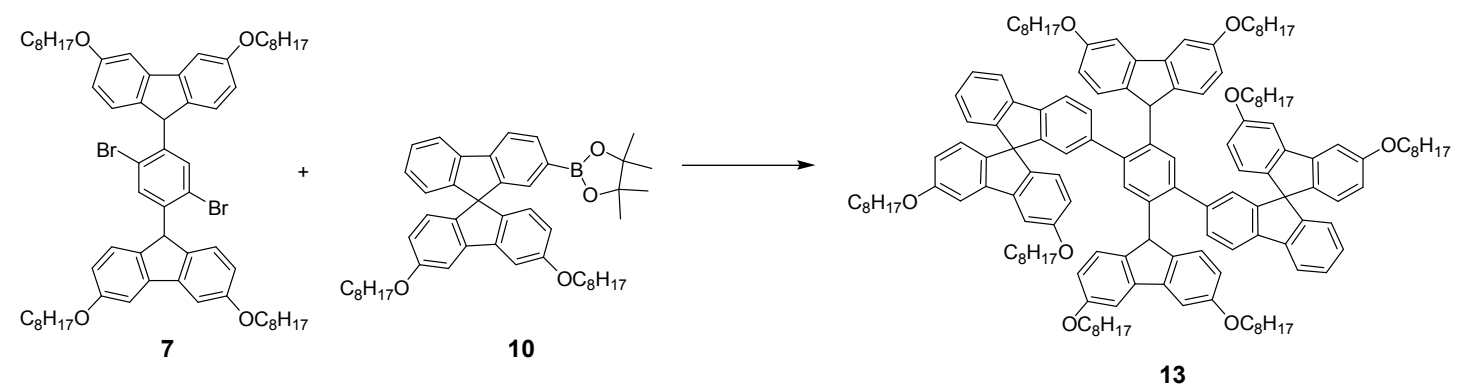

A mixture of 7 (50 mg, $0.046 \mathrm{mmol}$ ), 3',6'-bis(octyloxy)-9-9'-spirobisfluorenyl-2-boronic ester 10 (78 mg, $0.11 \mathrm{mmol}), \mathrm{NaHCO}_{3}(0.46 \mathrm{~g}, 5.5 \mathrm{mmol}), \mathrm{H}_{2} \mathrm{O}(4 \mathrm{~mL})$, and THF $(15 \mathrm{~mL})$ was carefully degassed before and after $\mathrm{Pd}\left(\mathrm{PPh}_{3}\right)_{4}(4 \mathrm{mg})$ was added. The mixture was heated to reflux and stirred under $\mathrm{N}_{2}$ for 24 hours. Water $(50 \mathrm{~mL})$ and $\mathrm{CH}_{2} \mathrm{Cl}_{2}(40 \mathrm{~mL})$ were added, the organic layer was separated, the aqueous layer was extracted with $\mathrm{CH}_{2} \mathrm{Cl}_{2}(50 \mathrm{~mL} \times 2)$, and the combined organic layers were dried over $\mathrm{Na}_{2} \mathrm{SO}_{4}$ and evaporated to dryness. The residue was chromatographically purified on silica gel eluting with $\mathrm{CH}_{2} \mathrm{Cl}_{2} /$ hexane $(1: 2, \mathrm{v} / \mathrm{v})$ to afford $\mathbf{1 3}$ as a colorless solid (91 mg, 95\%) ${ }^{1} \mathrm{H}$ NMR (400 MHz, $\left.\mathrm{CDCl}_{3}\right): \delta$ 7.72-7.66 (m, 4H), $7.37(\mathrm{~s}, 4 \mathrm{H}), 7.21$ (s, 4H), $7.15(\mathrm{~d}, 2 \mathrm{H}), 7.05(\mathrm{~s}, 2 \mathrm{H}) 7.01(\mathrm{t}, 2 \mathrm{H}), 6.70-6.68(\mathrm{~m}, 8 \mathrm{H}), 6.55(\mathrm{~d}, 4 \mathrm{H}) 6.42-6.39$ (broad, $8 \mathrm{H}) 6.26(\mathrm{~s}, 2 \mathrm{H}) 4.67(\mathrm{~s}, 2 \mathrm{H}) 3.98(\mathrm{t}, 8 \mathrm{H}), 3.90(\mathrm{t}, 8 \mathrm{H}) 1.81(\mathrm{~m}, 16 \mathrm{H}), 1.48(\mathrm{~m}, 16 \mathrm{H}), 1.49-1.31$ (broad, 64H), $0.90(\mathrm{t}, 24 \mathrm{H}) ;{ }^{13} \mathrm{C} \mathrm{NMR}\left(100 \mathrm{MHz}, \mathrm{CDCl}_{3}\right): \delta 159.9,159.2,150.1,150.0,143.5$, $142.9,142.6,141.9,141.7,141.5,140.7,138.4,129.4,128.8,128.3,128.1,126.3,125.2,124.6$, 120.5, 120.2, 114.8, 114.6, 106.4, 105.6, 68.8, 65.3, 48.9, 32.6, 30.2, 30.0, 26.8, 23.4, 14.8 . MALDI-TOF, m/z: calcd, 2060.9; found, $2060.8\left(\mathrm{M}^{+}\right)$

Synthesis and characterization of spiro-bridged ladder-type oligomer 3.

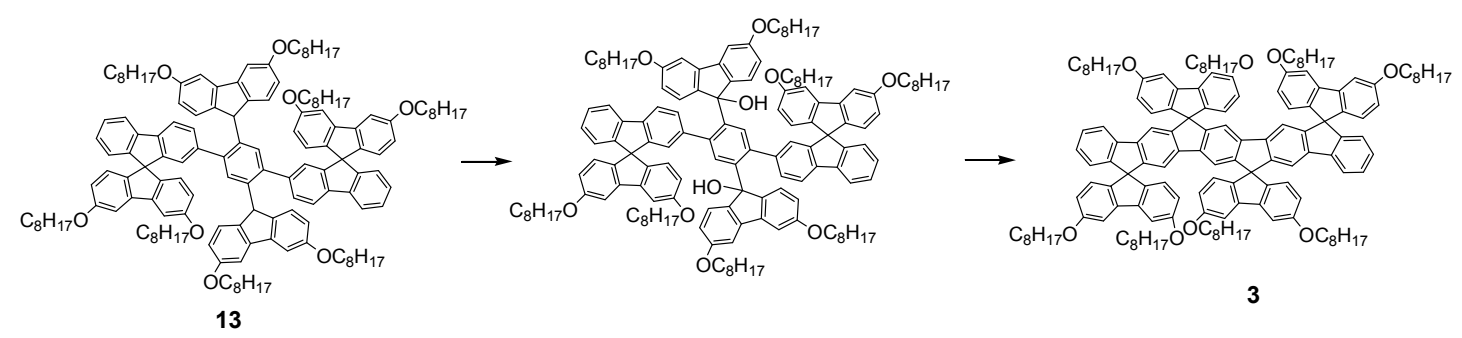

A mixture of 13 (0.15 g, $0.072 \mathrm{mmol})$, DMSO (32 mL), $t$-Butanol $(8 \mathrm{~mL})$, and KOH (3.5 g) was 
stirred vigorously for $12 \mathrm{~h}$ under oxygen atmosphere. Water $(50 \mathrm{~mL})$, acetic acid $(10 \mathrm{~mL})$, and $\mathrm{CH}_{2} \mathrm{Cl}_{2}(50 \mathrm{~mL})$ were added, the organic layer was separated, the aqueous layer was extracted with $\mathrm{CH}_{2} \mathrm{Cl}_{2}(50 \mathrm{~mL} \times 2)$, and the combined organic layers were washed with $(200 \mathrm{~mL} \times 3)$, dried over $\mathrm{Na}_{2} \mathrm{SO}_{4}$ and evaporated to dryness under reduced pressure. The residue was dissolved in dried $\mathrm{CH}_{2} \mathrm{Cl}_{2}(40 \mathrm{~mL})$, and $\mathrm{BF}_{3} \cdot \mathrm{Et}_{2} \mathrm{O}(0.5 \mathrm{~mL})$ was added dropwise to this mixture. After stirring for 10 min, $10 \mathrm{~mL}$ of ethanol was added, the organic layer was washed with water $(20 \mathrm{~mL} \times 3)$. After removal of the solvent, the residue was chromatographically purified on silica gel eluting with $\mathrm{CH}_{2} \mathrm{Cl}_{2} /$ hexane $(1: 2, \mathrm{v} / \mathrm{v})$ to afford $3(0.14 \mathrm{~g} 93 \%)$ as a colorless solid. ${ }^{1} \mathrm{H} \mathrm{NMR}\left(400 \mathrm{MHz}, \mathrm{CDCl}_{3}\right)$ : $\delta 7.42(\mathrm{~d}, 2 \mathrm{H}), 7.35(\mathrm{~s}, 4 \mathrm{H}), 7.28(\mathrm{~s}, 4 \mathrm{H}), 7.14(\mathrm{t}, 2 \mathrm{H}), 7.02(\mathrm{~s}, 2 \mathrm{H}), 6.95(\mathrm{t}, 2 \mathrm{H}), 6.75(\mathrm{~s}, 2 \mathrm{H}), 6.73$ (s, 2H), 6.69-6.54 (m, 18H), 4.06-3.99 (m, 16H), $1.82(\mathrm{~m}, 16 \mathrm{H}), 1.49(\mathrm{~m}, 16 \mathrm{H}), 1.34-1.26$ (broad, 64), $0.90(\mathrm{t}, 24 \mathrm{H}) ;{ }^{13} \mathrm{C} \mathrm{NMR}\left(100 \mathrm{MHz}, \mathrm{CDCl}_{3}\right): \delta 160.0,160.0,150.1,150.0,149.6,143.6,143.6$, $142.4,142.2,142.2,142.1,141.8,141.8,127.9,125.5,124.2,120.4,115.9,115.7,115.0,106.5$, 106.4, 68.9, 68.9, 65.0, 64.9, 32.5, 30.4, 30.1, 30.0, 26.8, 26.8, 23.4, 14.8. MALDI-TOF, m/z: calcd, 2056.9; found, $2056.3\left(\mathrm{M}^{+}\right)$.

\section{Synthesis and characterization of compound 15.}

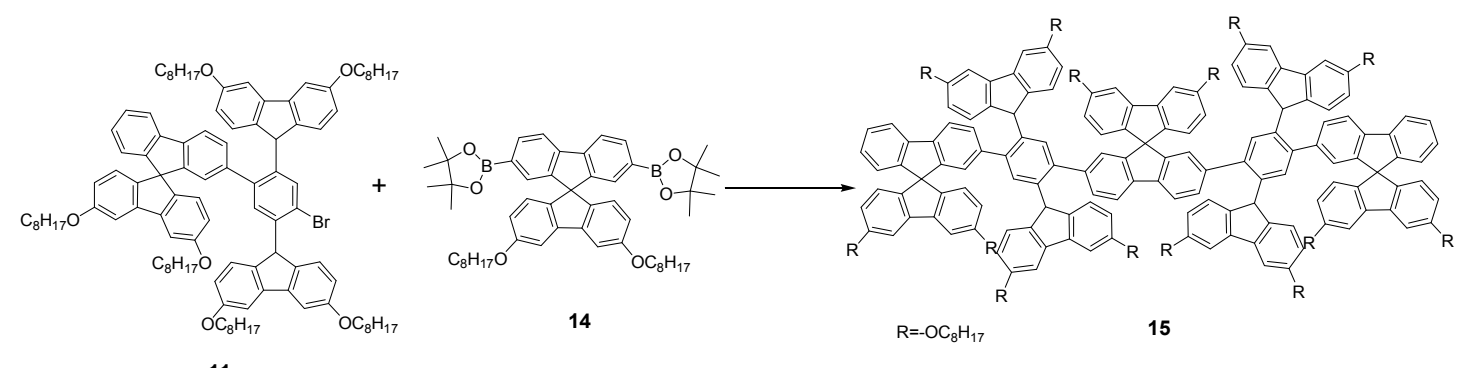

A mixture of 11 (96 mg, $0.061 \mathrm{mmol}$ ), 3',6'-bis(octyloxy)-9-9'-spirobisfluorenyl-2,7-bisboronic ester $(23 \mathrm{mg}, 0.028 \mathrm{mmol}), \mathrm{NaHCO}_{3}(0.25 \mathrm{~g}, 3.0 \mathrm{mmol}), \mathrm{H}_{2} \mathrm{O}(4 \mathrm{~mL})$, and THF $(15 \mathrm{~mL})$ was carefully degassed before and after $\mathrm{Pd}\left(\mathrm{PPh}_{3}\right)_{4}(4 \mathrm{mg})$ was added. The mixture was heated to reflux and stirred under $\mathrm{N}_{2}$ for $24 \mathrm{~h}$. Water $(40 \mathrm{~mL})$ and $\mathrm{CH}_{2} \mathrm{Cl}_{2}(40 \mathrm{~mL})$ were added, the organic layer was separated, the aqueous layer was extracted with $\mathrm{CH}_{2} \mathrm{Cl}_{2}(40 \mathrm{~mL} \times 2)$, and the combined organic layers were dried over $\mathrm{Na}_{2} \mathrm{SO}_{4}$ and evaporated to dryness. The residue was chromatographically purified on silica gel eluting with $\mathrm{CH}_{2} \mathrm{Cl}_{2} /$ hexane $(1: 3, \mathrm{v} / \mathrm{v})$ to afford $\mathbf{1 5}$ as a colorless solid (86 mg, 87\%) ${ }^{1} \mathrm{H}$ NMR (400 MHz, $\mathrm{CDCl}_{3}$ ): $\delta$ 7.71-7.57 (m, 6H), 7.28-7.24 (m, 8H), $7.20(\mathrm{~s}, 6 \mathrm{H}), 7.13-7.02(\mathrm{~m}, 16 \mathrm{H}), 6.69-6.66(\mathrm{~m}, 12 \mathrm{H}) 6.54(\mathrm{broad} 8 \mathrm{H}), 6.40$ (broad, 6H), 6.26-6.12 (broad, 6H) 4.63 (s, 2H) 4.61 (s, 2H) 3.97-3.77 (broad, 28H), 1.78 (broad, 28H), 1.47-1.31 (broad, 140H), 0.91 (broad, 42H); ${ }^{13} \mathrm{C} \mathrm{NMR} \mathrm{(100} \mathrm{MHz,} \mathrm{CDCl}_{3}$ ): $\delta 159.2,159.1,158.5,149.3,149.3,142.7$, 142.6, 142.2, 141.9, 141.2, 141.0, 140.8, 140.7, 140.6, 140.0, 137.7, 137.6, 128.7, 128.0, 127.5, 127.4, 125.5, 124.4, 123.9, 119.7, 119.5, 114.0, 113.9, 105.7, 104.9, 68.1, 64.6, 48.1, 31.9, 31.8, 29.6, 29.5, 29.4, 29.3, 26.1, 22.7, 14.1. MALDI-TOF, m/z: calcd, 3546.3; found, $3549.7\left(\mathrm{M}^{+}\right)$

\section{Synthesis and characterization of spiro-bridged ladder-type oligomer 4}




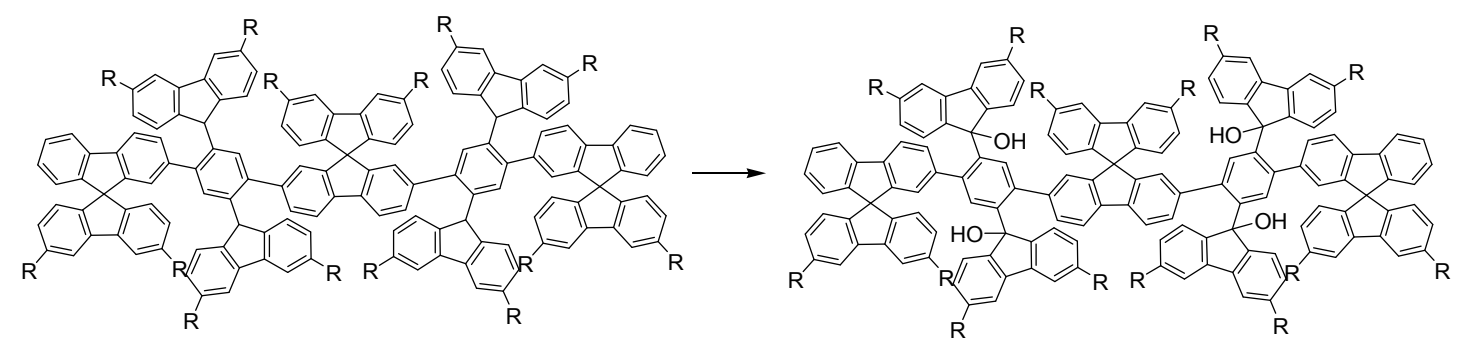

15

$\mathrm{R}=-\mathrm{OC}_{8} \mathrm{H}_{17}$

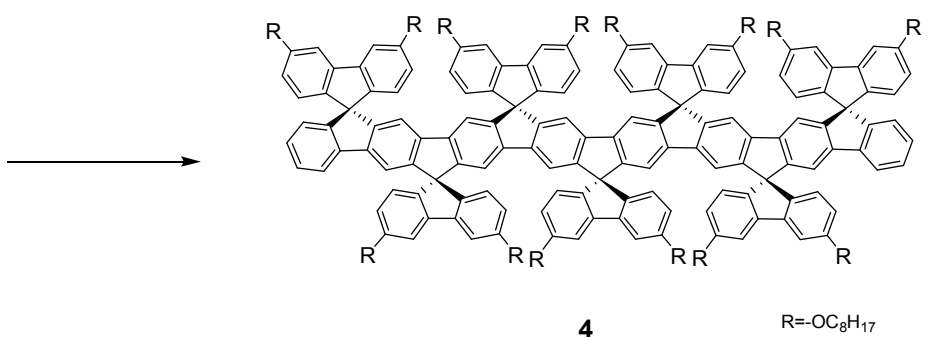

A mixture of 15 (40 mg, $0.011 \mathrm{mmol}), \mathrm{KOH}$ ( $30 \mathrm{mg}, 0.75 \mathrm{mmol})$, benzyltriethylammonium chloride (50 mg, $0.22 \mathrm{mmol})$, and pyridine $(10 \mathrm{~mL})$ was degassed before hydrogen peroxide $(0.02 \mathrm{~mL})$ in pyridine $(0.5 \mathrm{~mL})$ was added. The mixture was heated to $40{ }^{\circ} \mathrm{C}$ and stirred for $10 \mathrm{~min}$. Pyridine was removed under reduced pressure, the residual was filtered through a short pad of silica gel eluting with $\mathrm{CH}_{2} \mathrm{Cl}_{2} /$ ether $(10: 1, \mathrm{v} / \mathrm{v})$ to afford the intermediate tetraol. The tetraol was dissolved in $50 \mathrm{~mL}$ of dried $\mathrm{CH}_{2} \mathrm{Cl}_{2}, \mathrm{BF}_{3} \cdot \mathrm{Et}_{2} \mathrm{O}(0.5 \mathrm{~mL})$ was added dropwise, and the resulted mixture was stirred for $10 \mathrm{~min}$. Ethanol $(10 \mathrm{~mL})$ was added, the organic layer was washed with water $(30 \mathrm{~mL} \times 3)$ and dried over $\mathrm{Na}_{2} \mathrm{SO}_{4}$. After removal of the solvent, the crude product was chromatographically purified on silica gel eluting with $\mathrm{CH}_{2} \mathrm{Cl}_{2} /$ hexane $(1: 3, \mathrm{v} / \mathrm{v})$ to afford 4 as a pale yellow solid. ( $3 \mathrm{mg}$, 8\%) ${ }^{1} \mathrm{H}$ NMR (400 MHz, $\mathrm{CDCl}_{3}$ ): $\delta$ 7.52-7.23 (broad, 6H), 7.09-6.79 (broad, 24H), 6.65-6.51 (broad, 30), 4.17-3.96 (broad, 28H), 1.91-1.85 (broad, 28H), 1.50-1.26 (broad, 140H), 0.90 (broad, 42H). MALDI-TOF, m/z: calcd, 3538.3; found, $3540.3\left(\mathrm{M}^{+}\right)$

\section{References}

1) Lee, H.; Oh, J.; Chu, Y.; Lee, J.-I.; Kim, S. H.; Yang Y. S.; Kim, G. H.; Do, L. M.; Lee, J.; Park, Y. Tetrahedron, 2003, 59, 2773.

2) Xia, C.; Advincula, R. C. Macromolecules, 2001, 34, 6922.

3) Tolman, C. A.; Seidel, W. C.; Gerlach, D. H. J. Am. Chem. Soc. 1972, 94, 2669. 


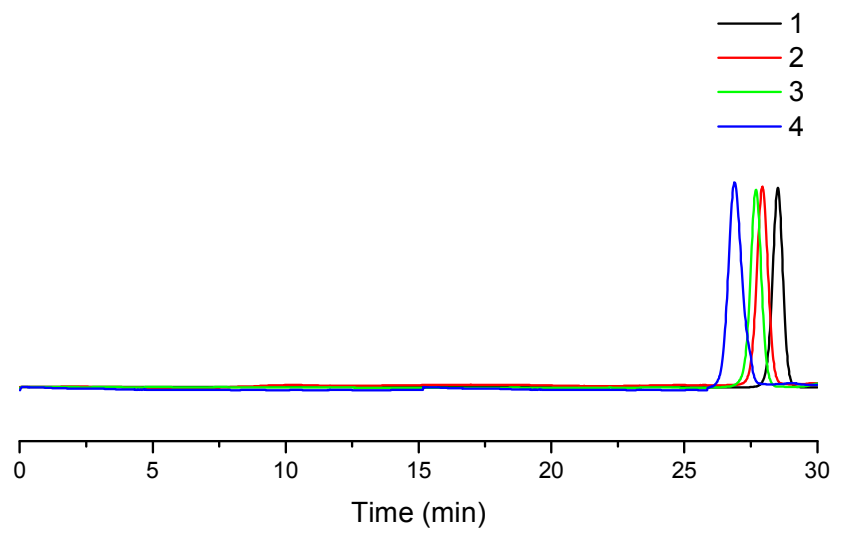

Figure S1. The GPC elution traces of spiro-bridged ladder-type oligo-p-phenylenes 1-4.

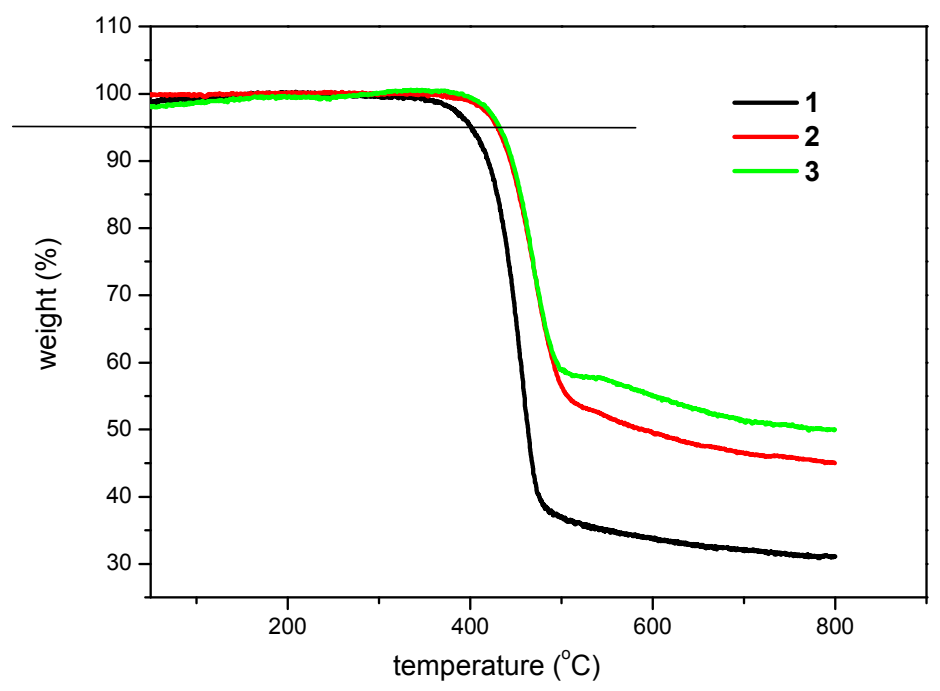

Figure S2. TGA traces of ladder-type oligomers 1-3. 


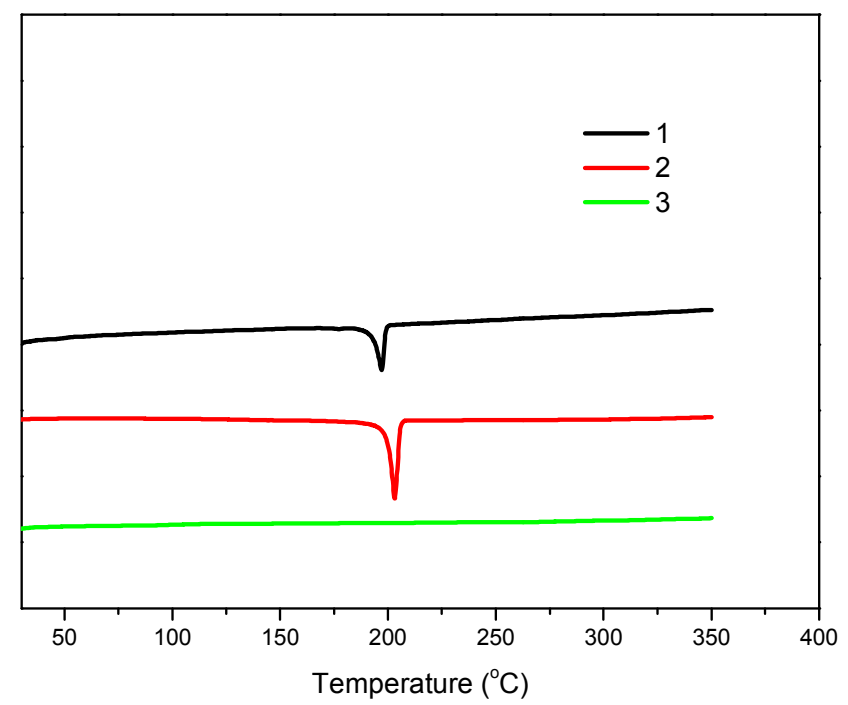

Figure S3. DSC traces of ladder-type oligomers 1-3.

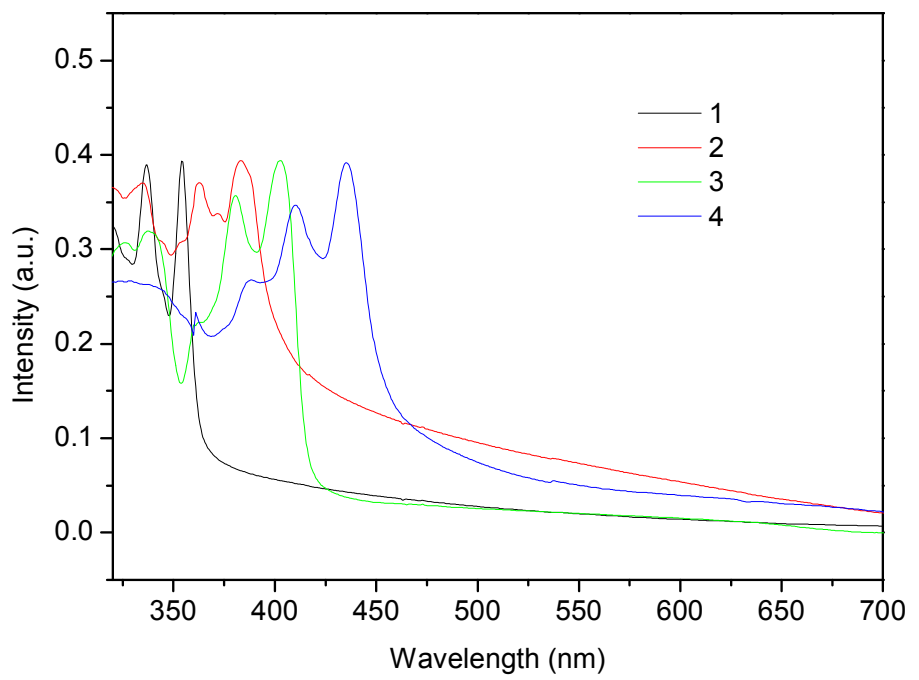

Figure S4. The film UV-vis absorption spectra of ladder-type oligomers 1-4. 


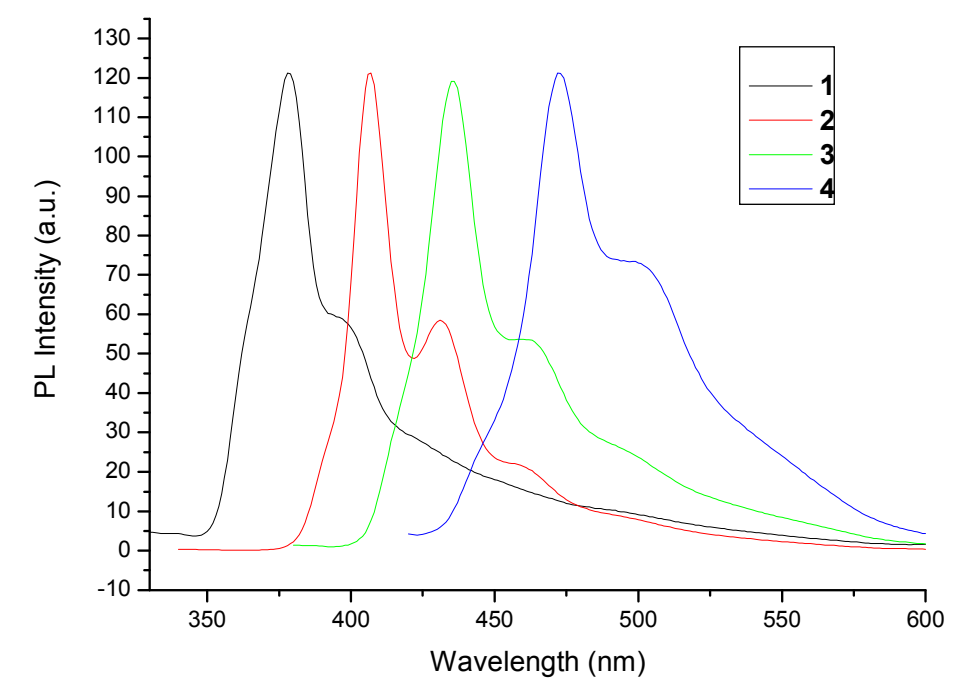

Figure S5. The film PL spectra of ladder-type oligomers 1-4.
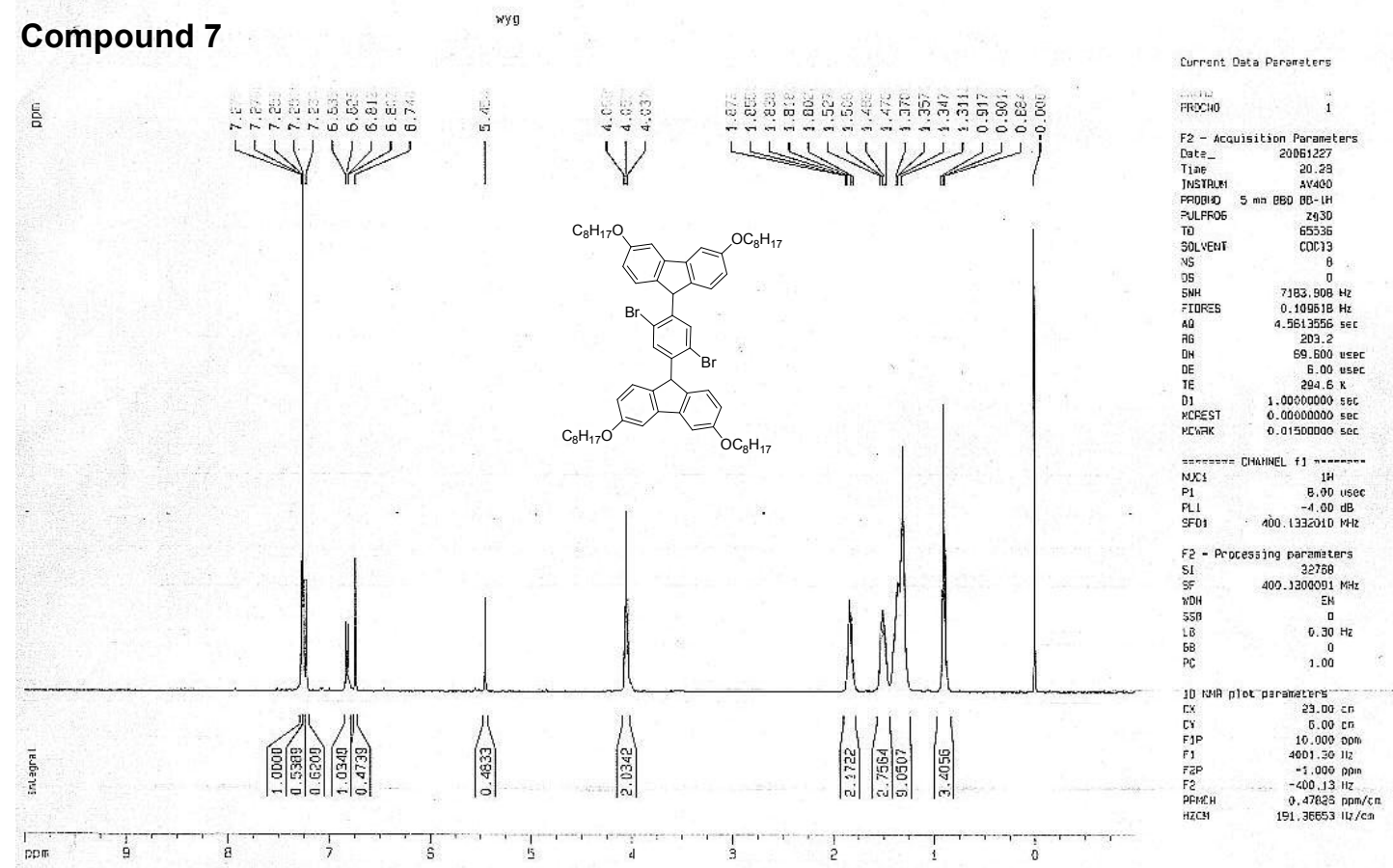


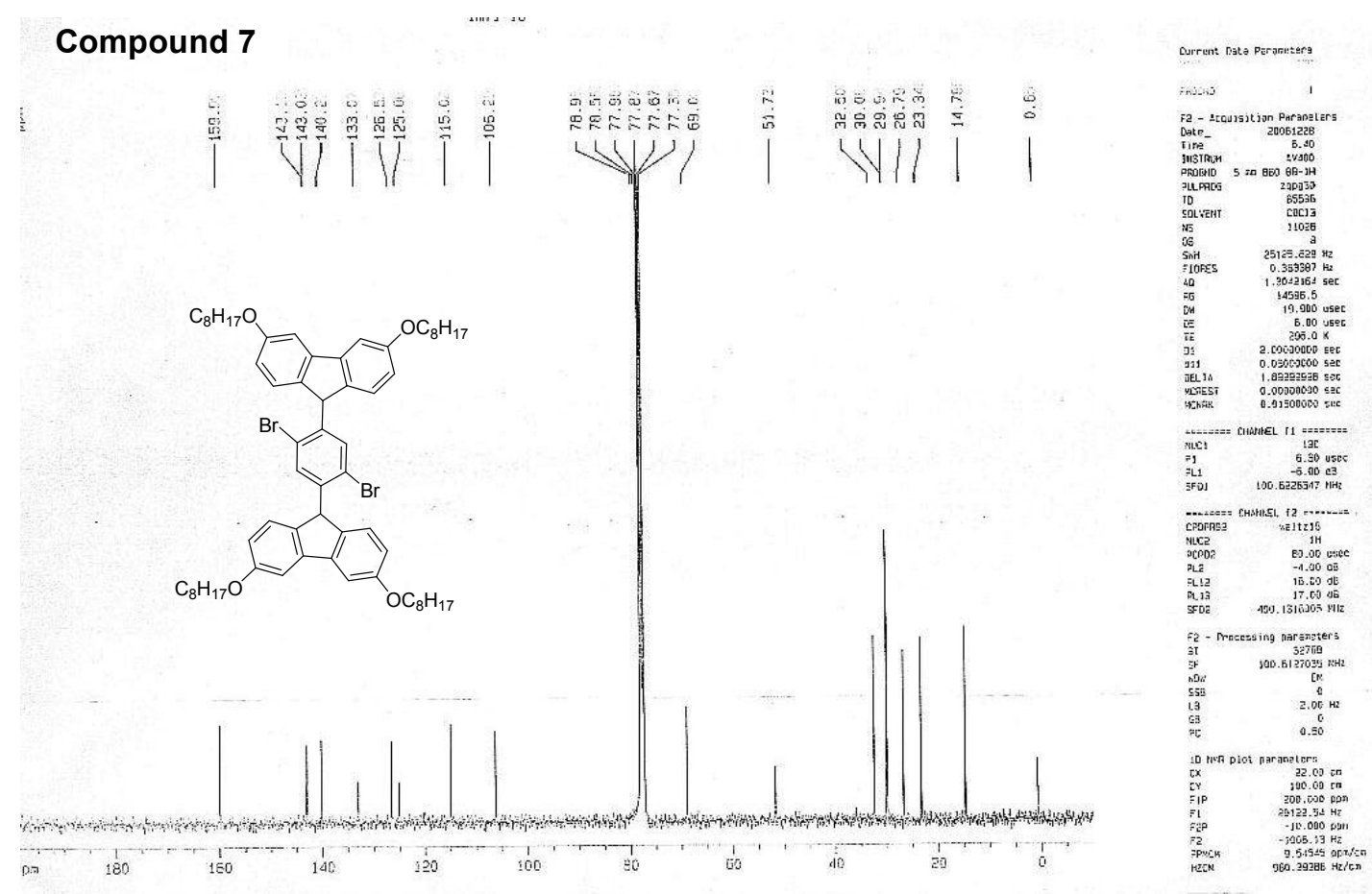

\section{Compound 8}
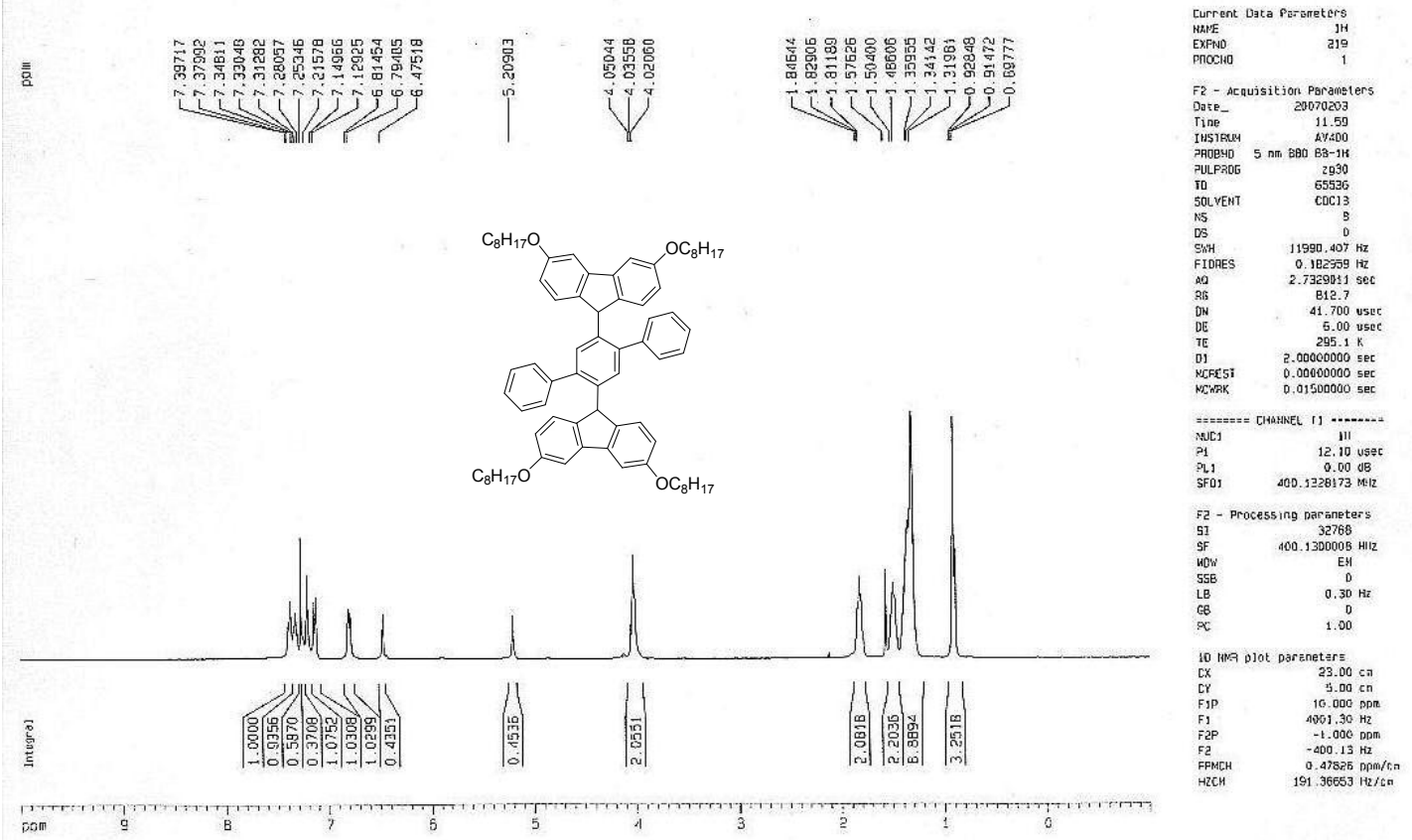


\section{Compound 8}

wyg-2befora-ax

镸

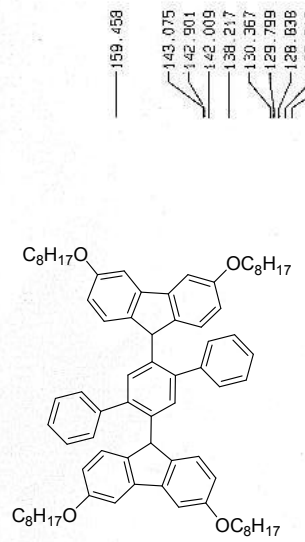

(a)

DD?

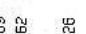

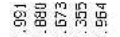

勿

W|

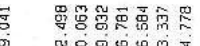

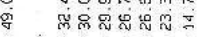

WVII

IV?

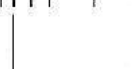

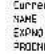

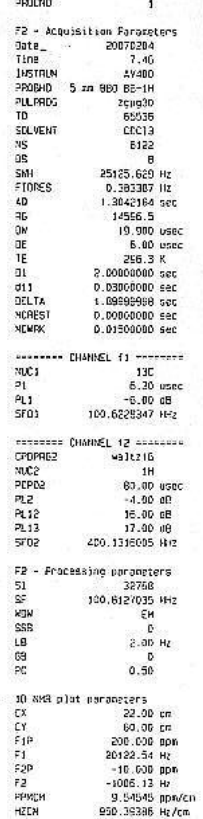

\section{Compound 8}

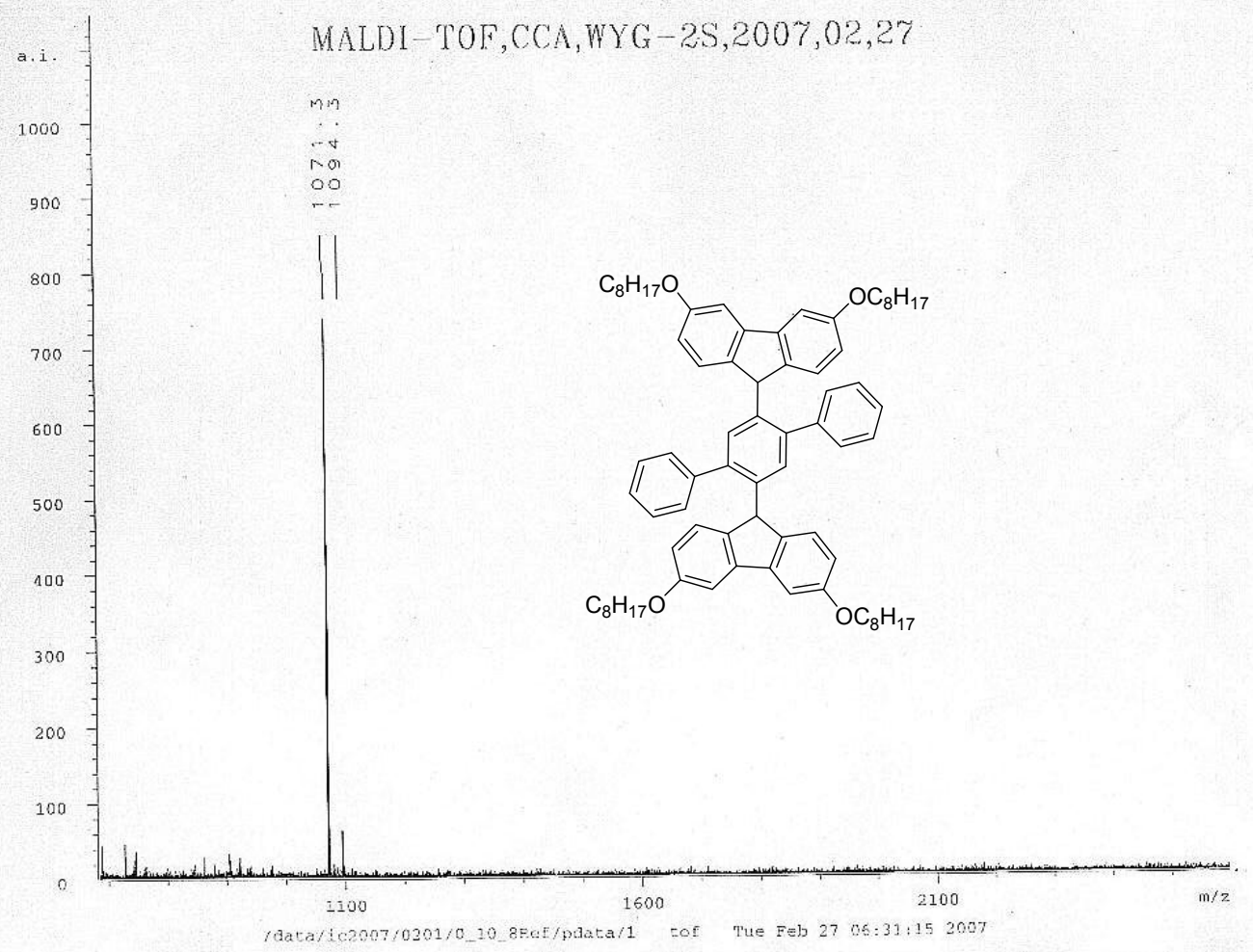




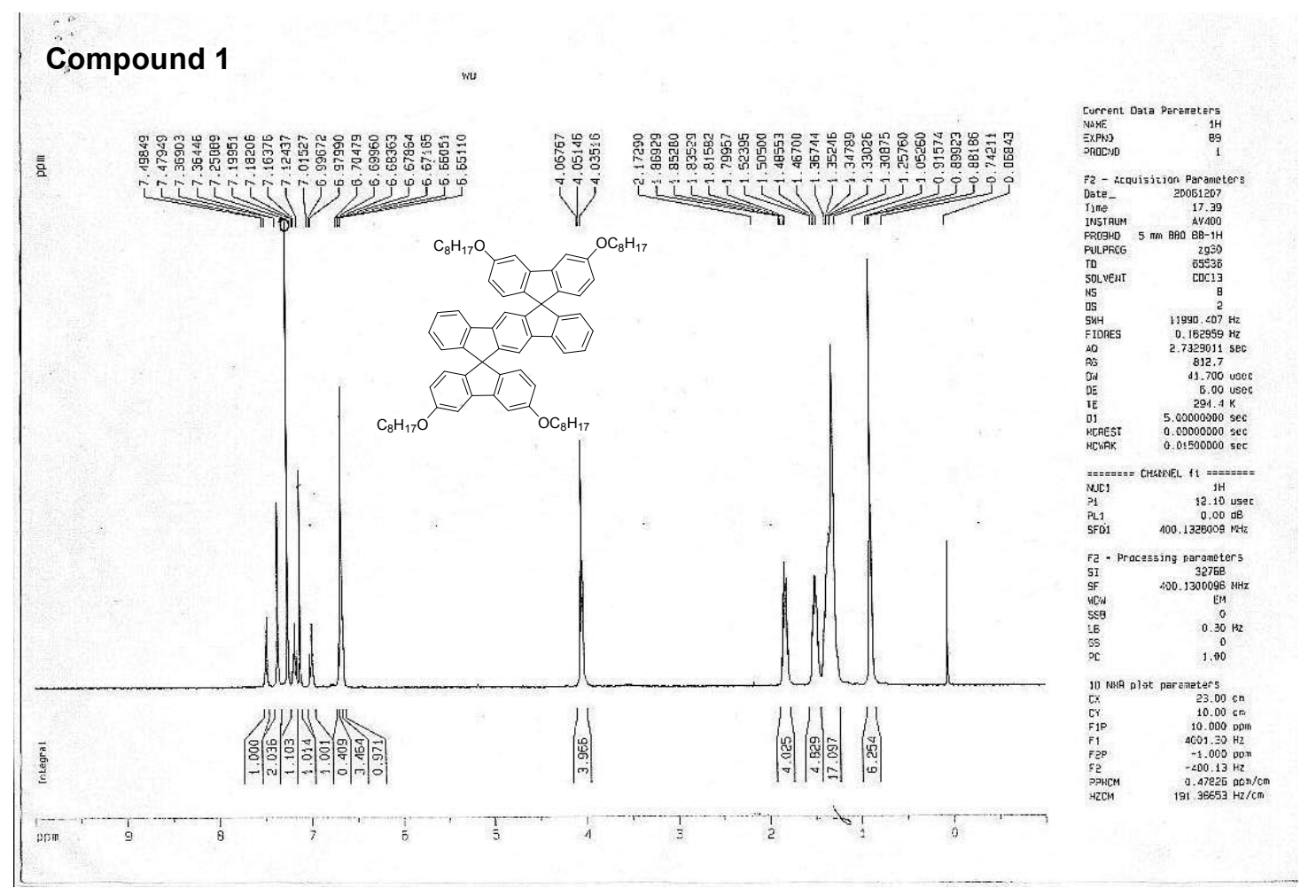

\section{Compound 1}

wyy

言

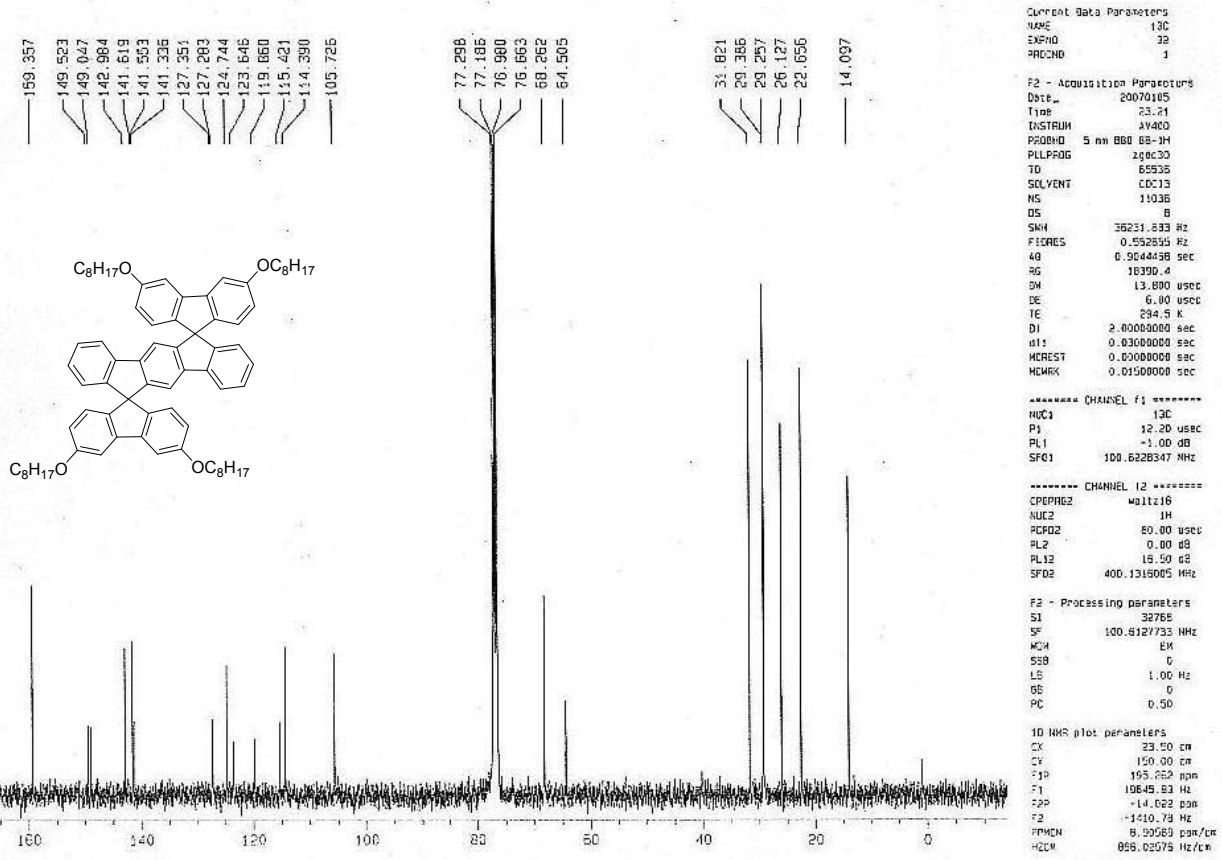



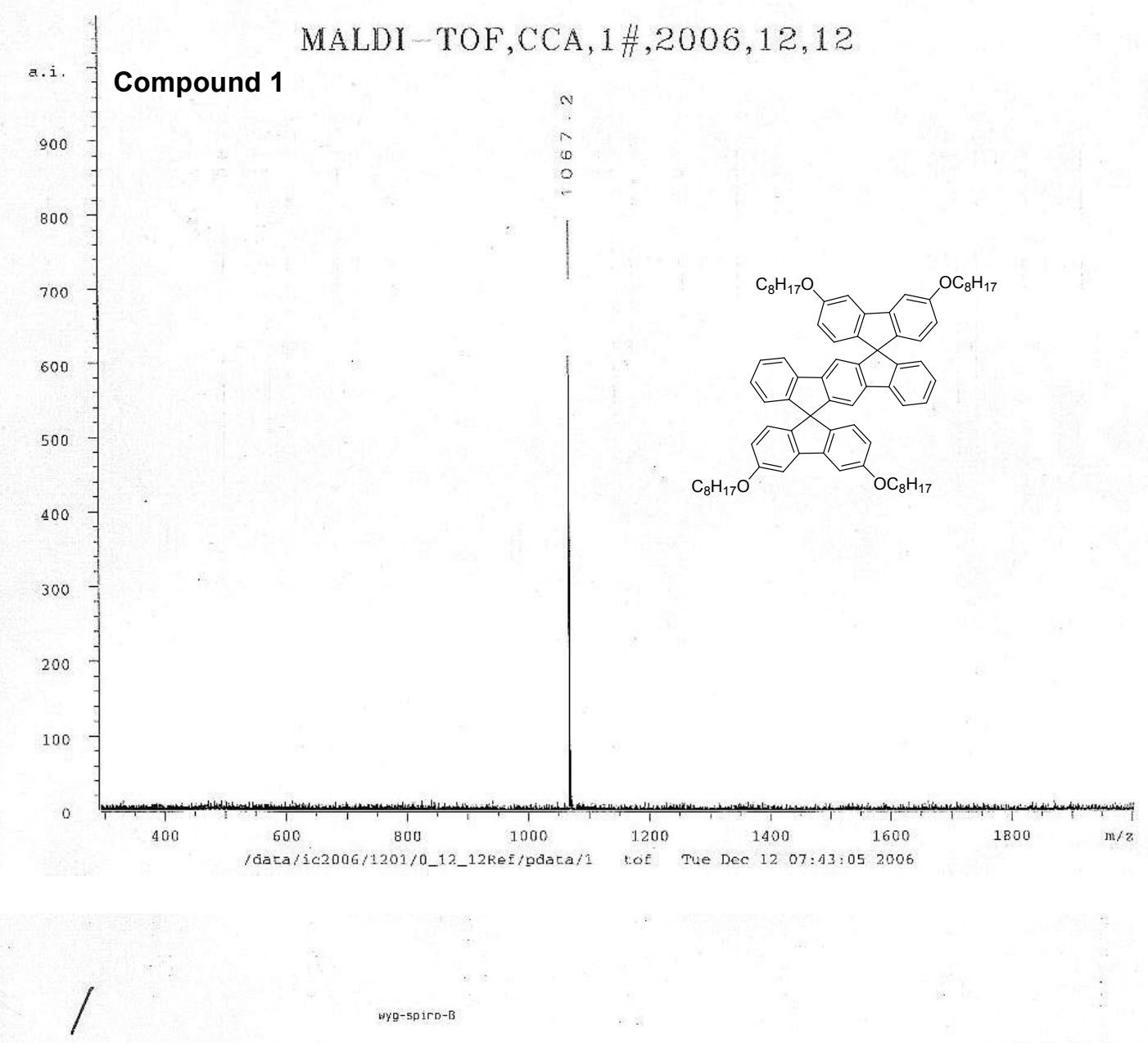

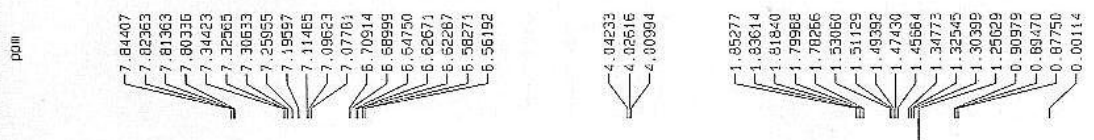

\section{Compound 10}
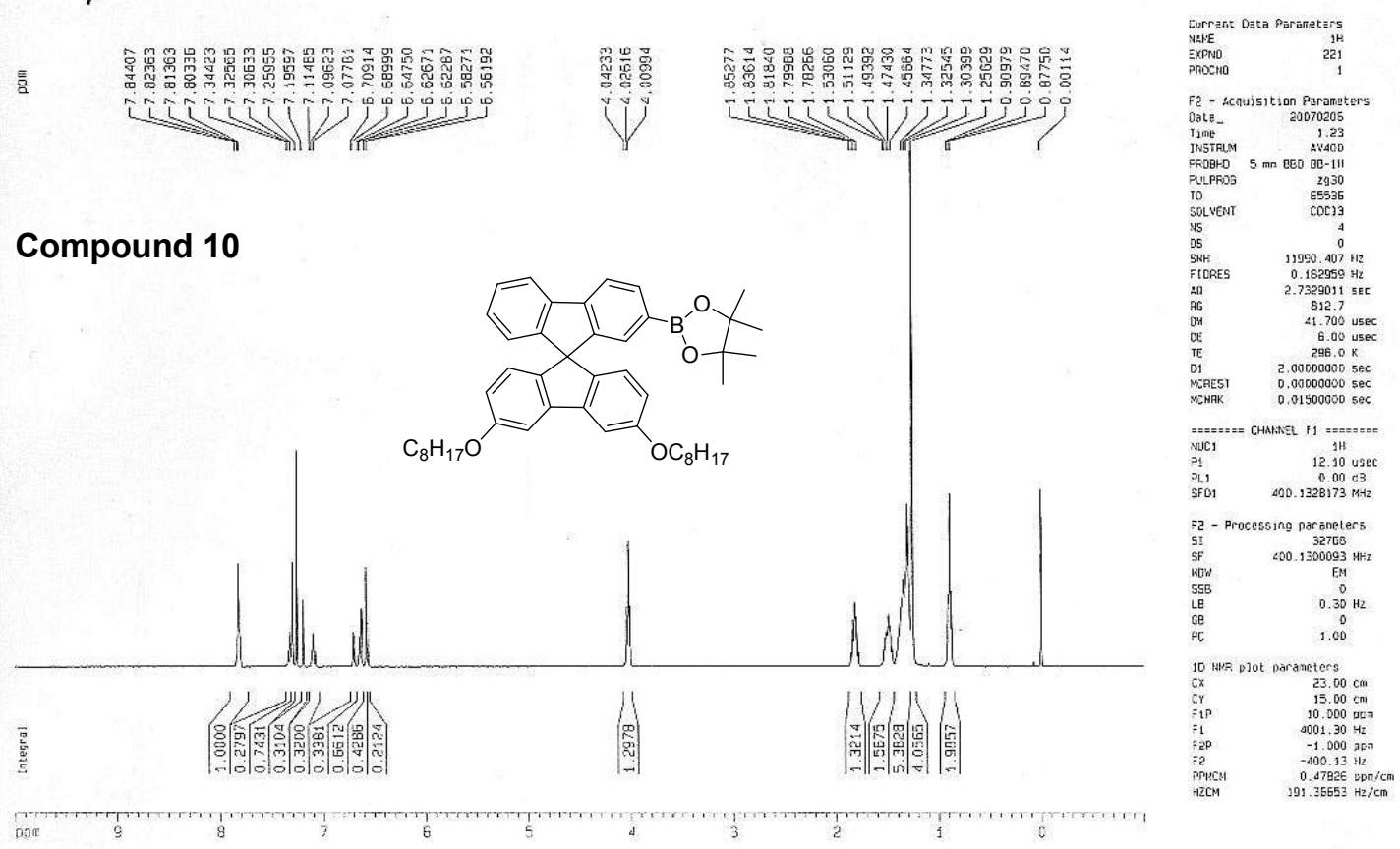


\section{Compound 11}

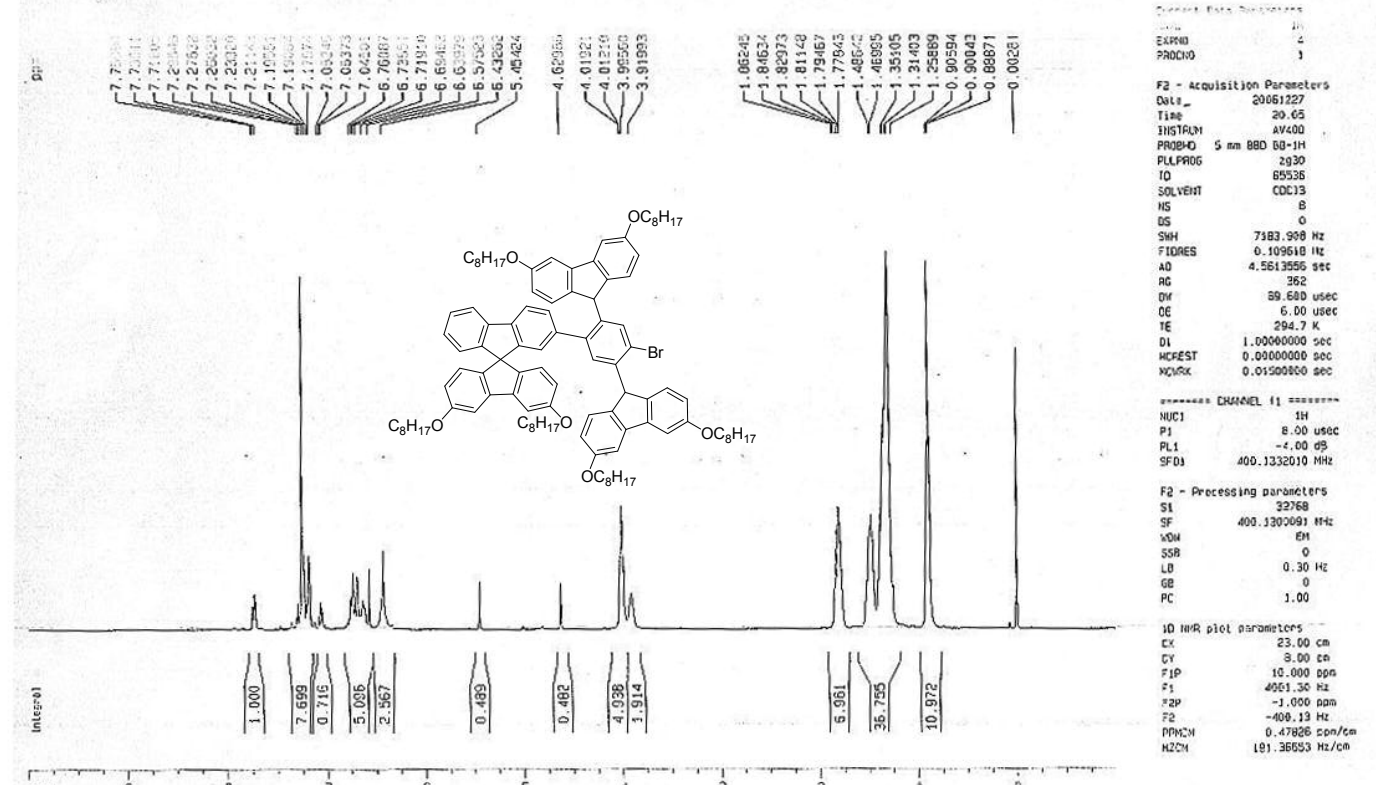

\section{Compound 11}

wyg-33r

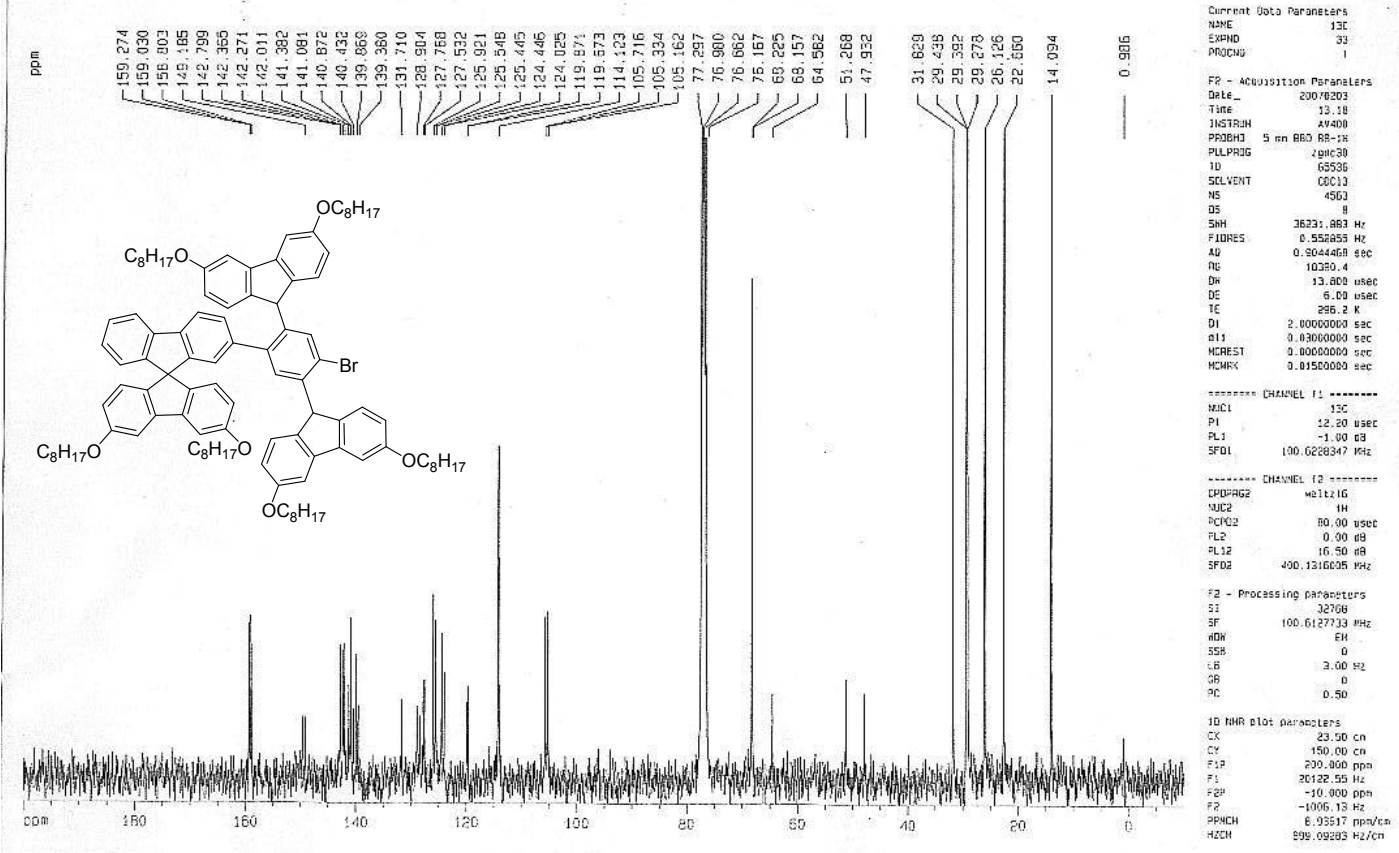



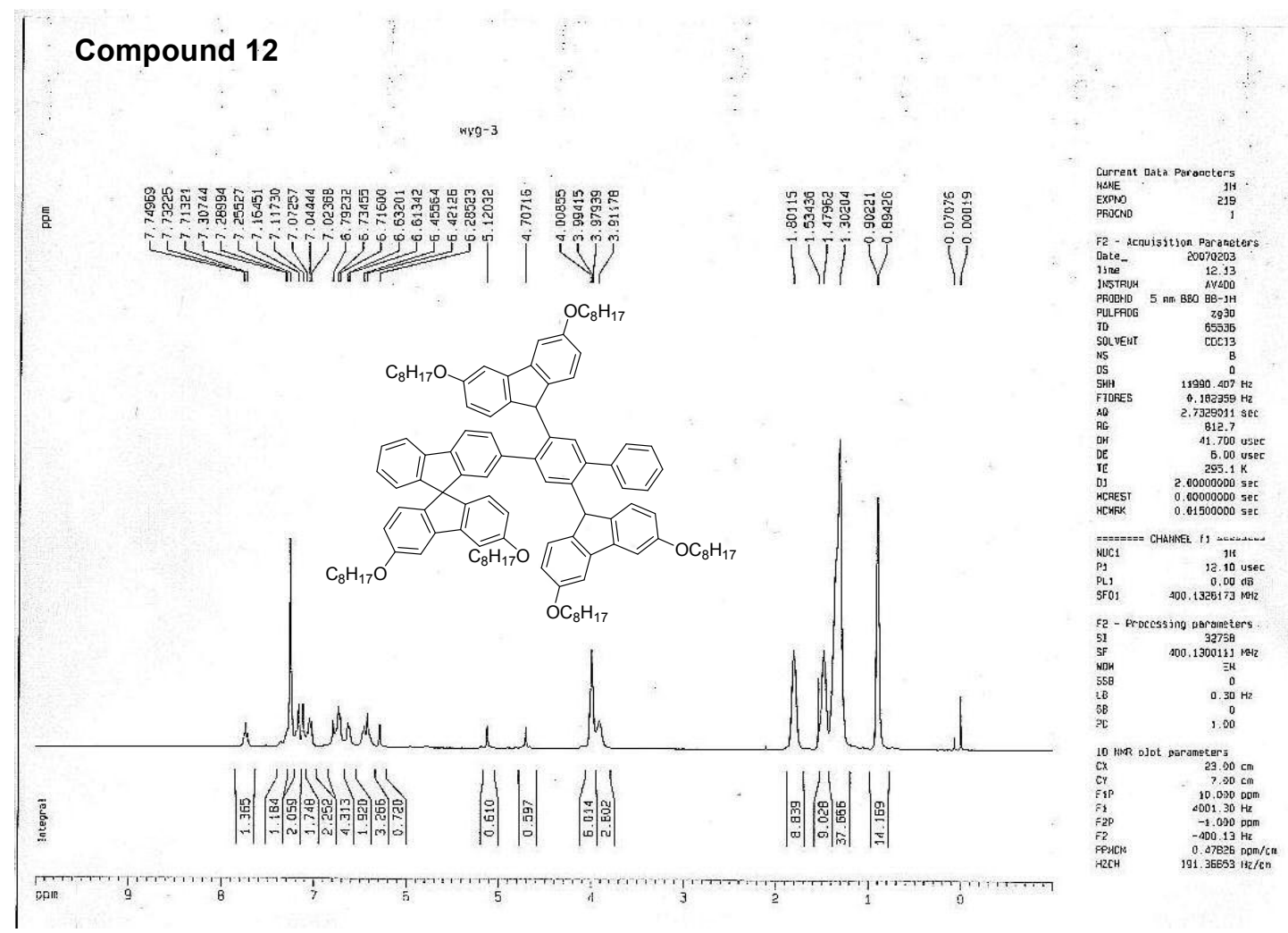

\section{Compound 12}

言

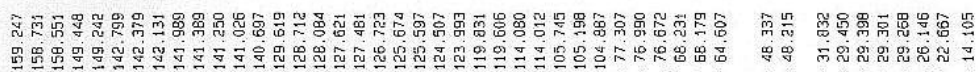

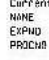
tist
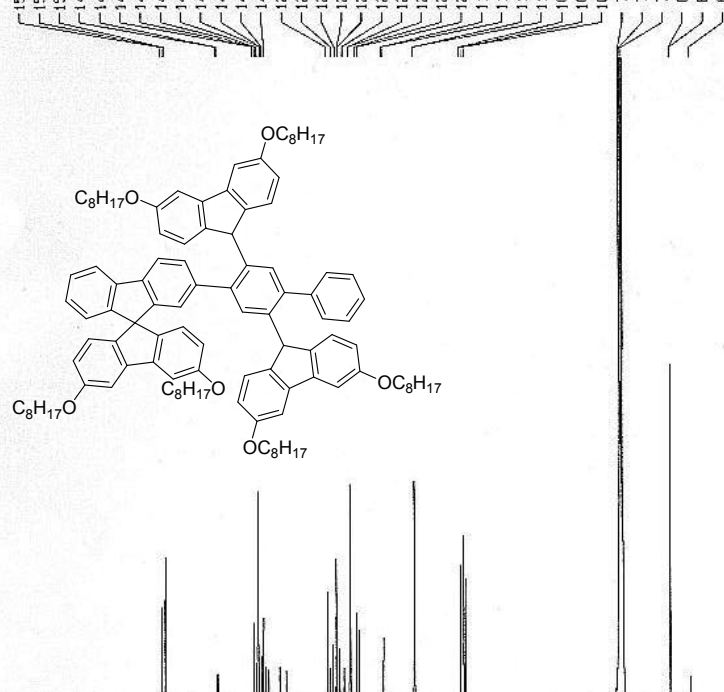

Y

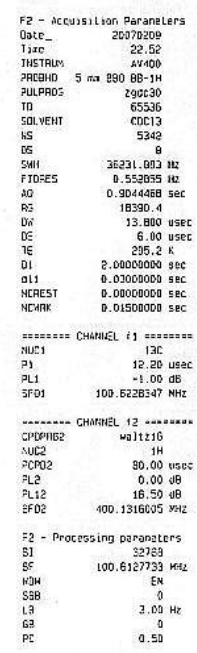

Ant

190 


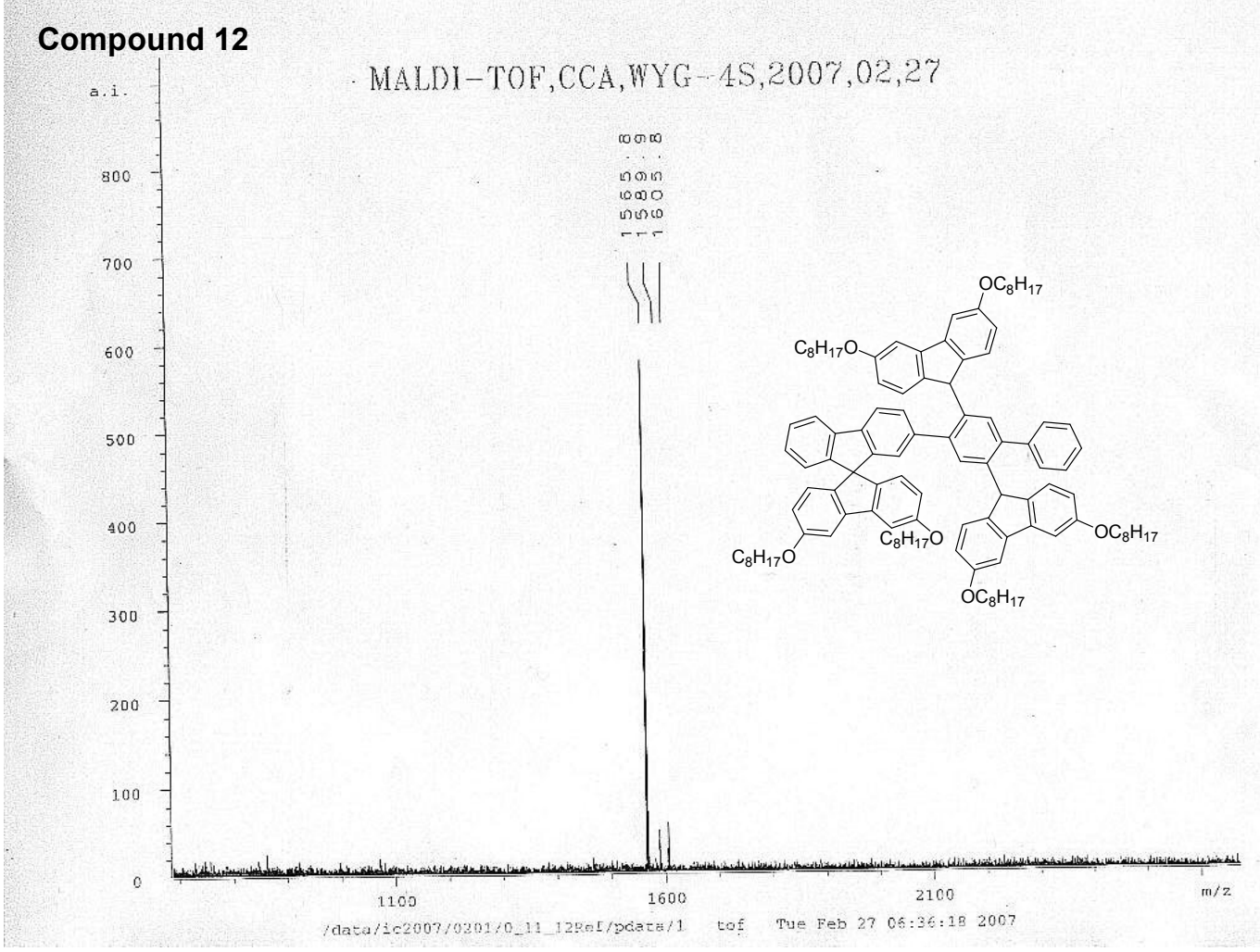

Compound 2

ryg-3s

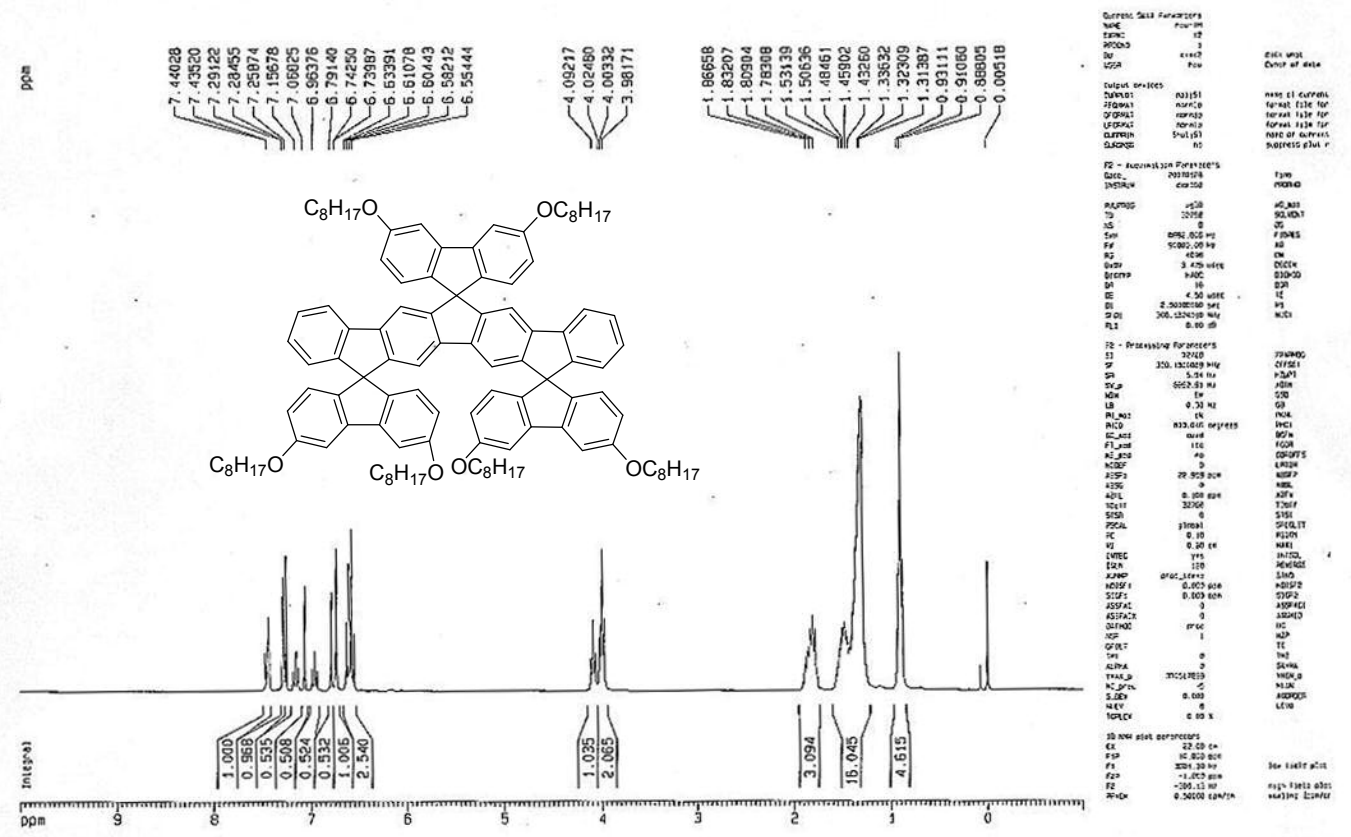



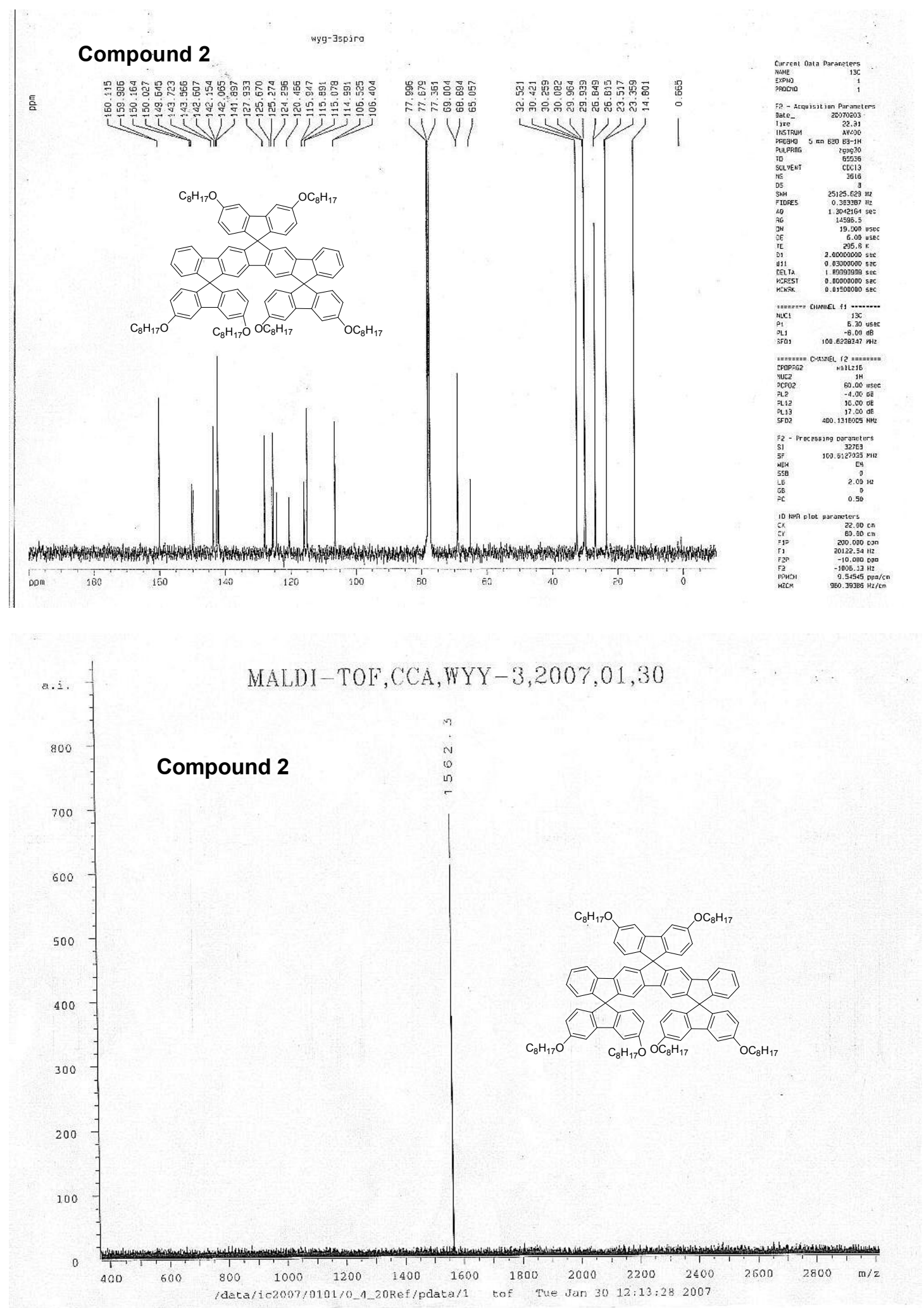

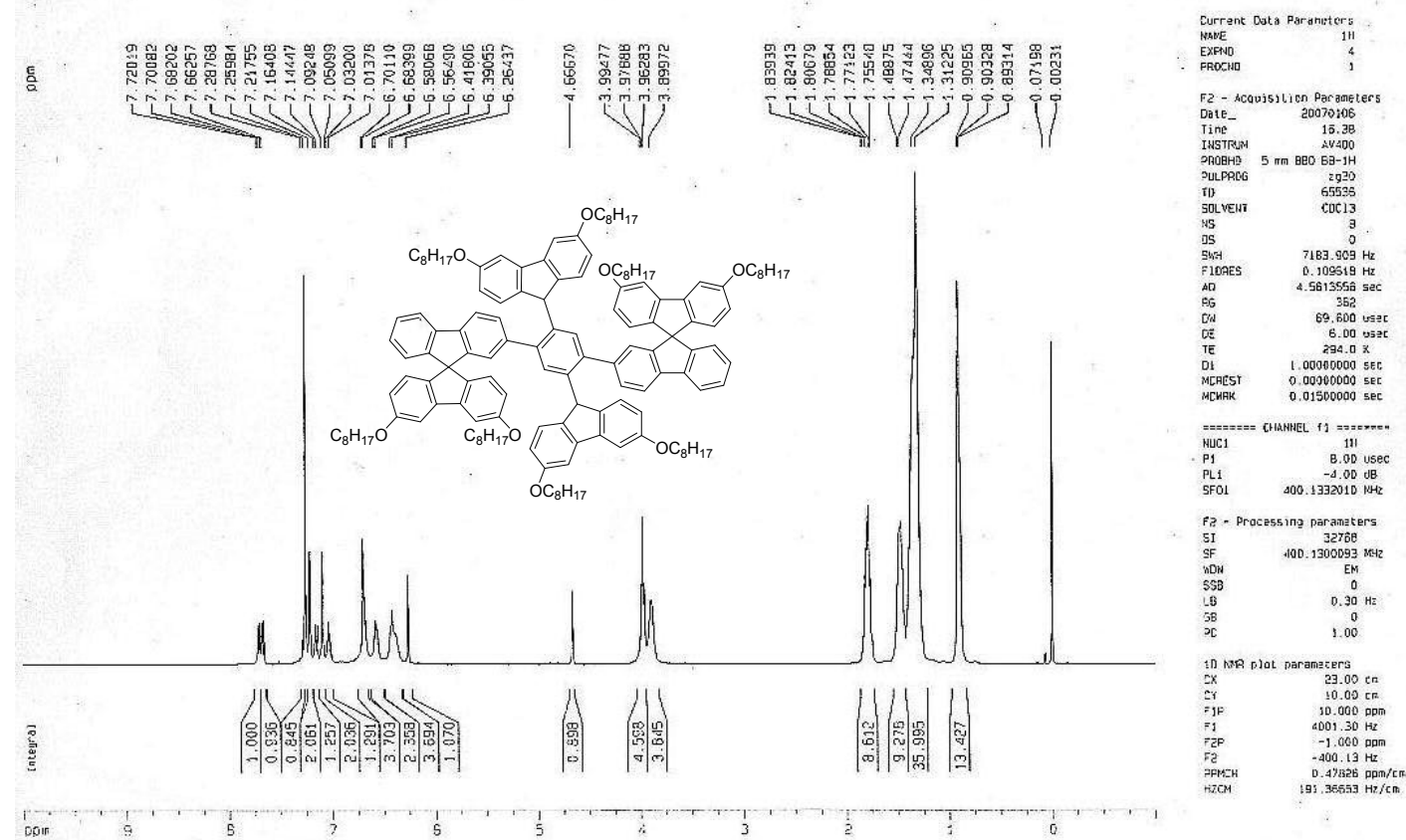

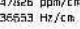

\section{Compound 13}
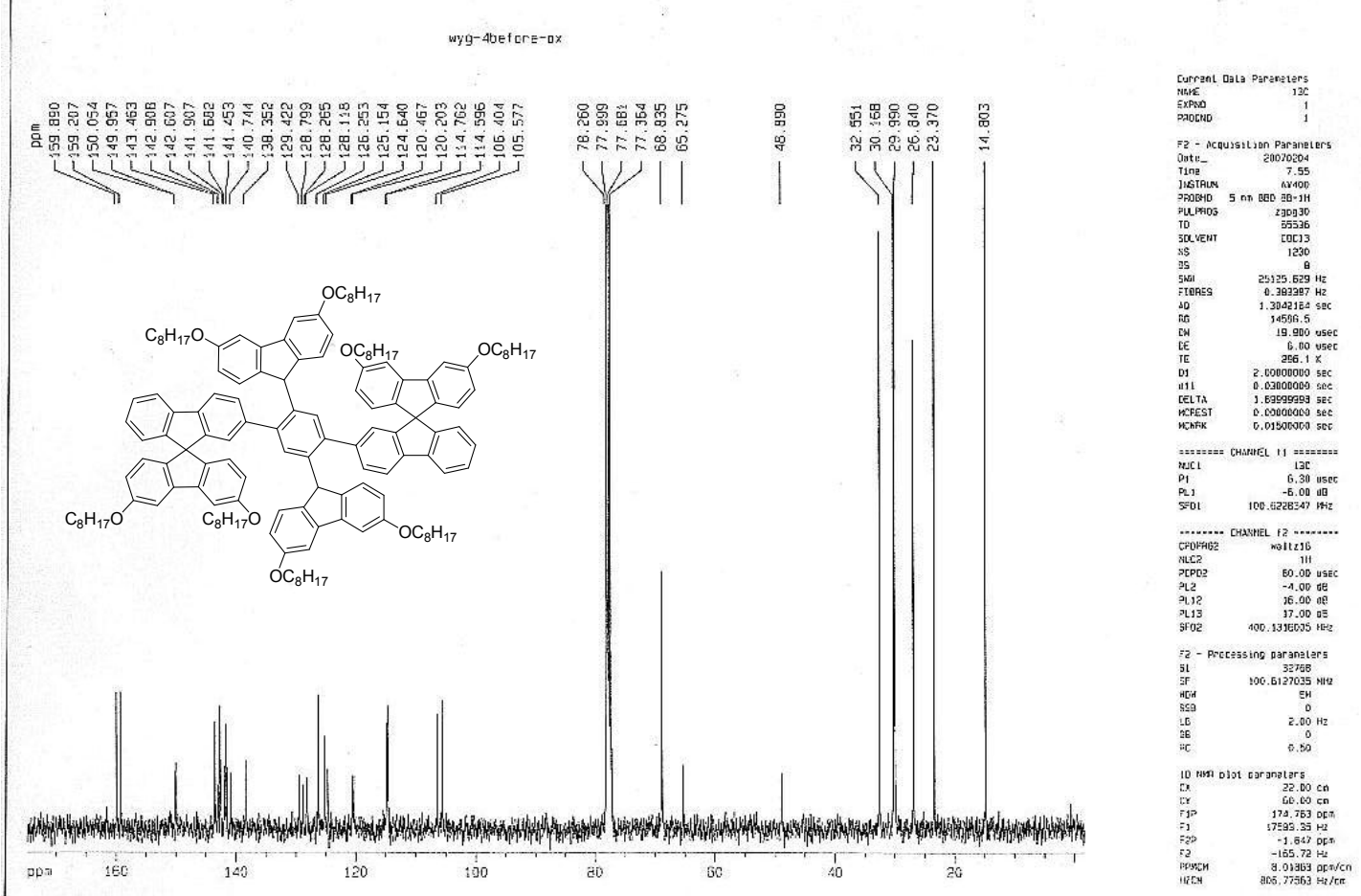


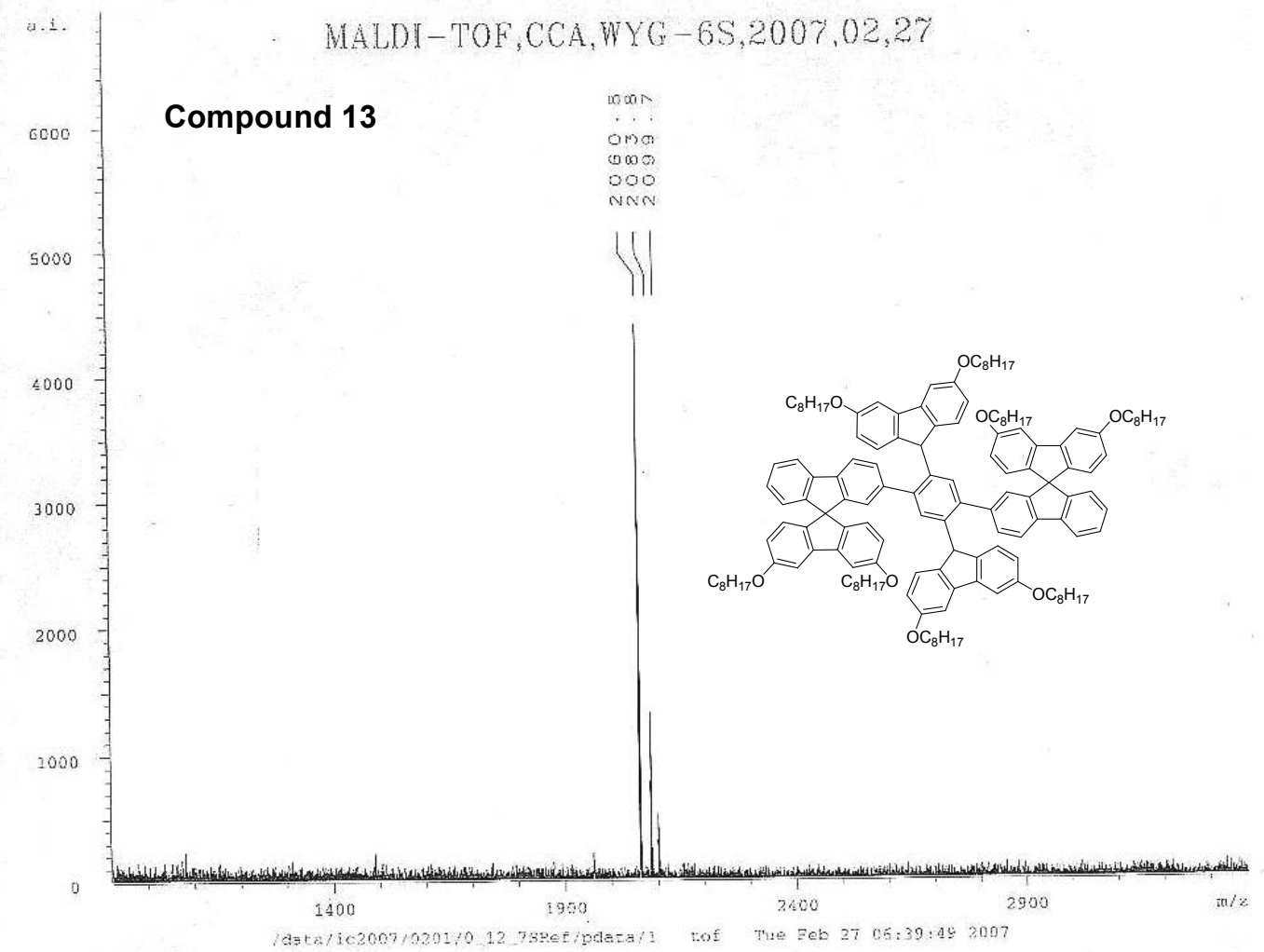

2haoning-50
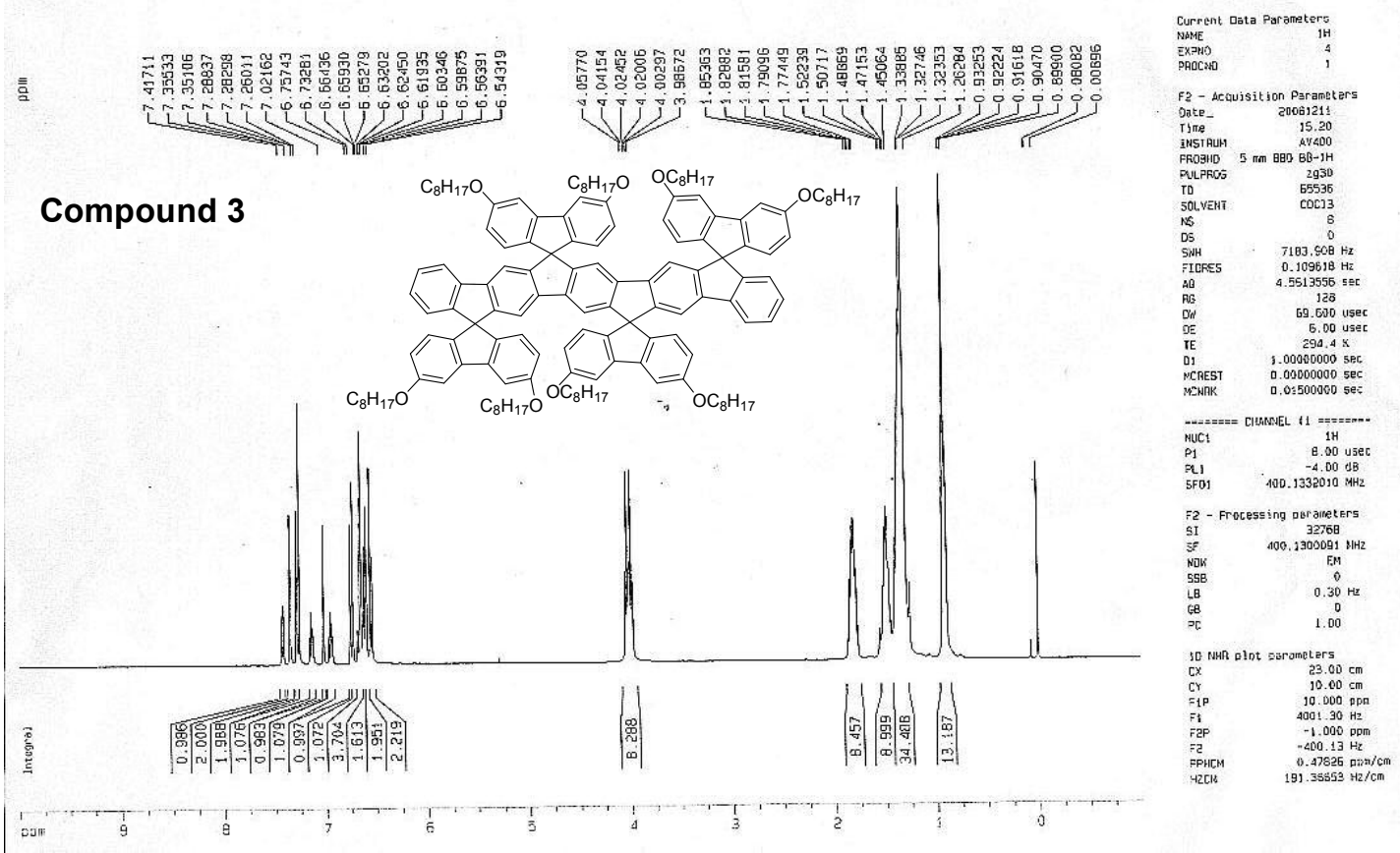


\section{Compound 3}

1อง $1-18$
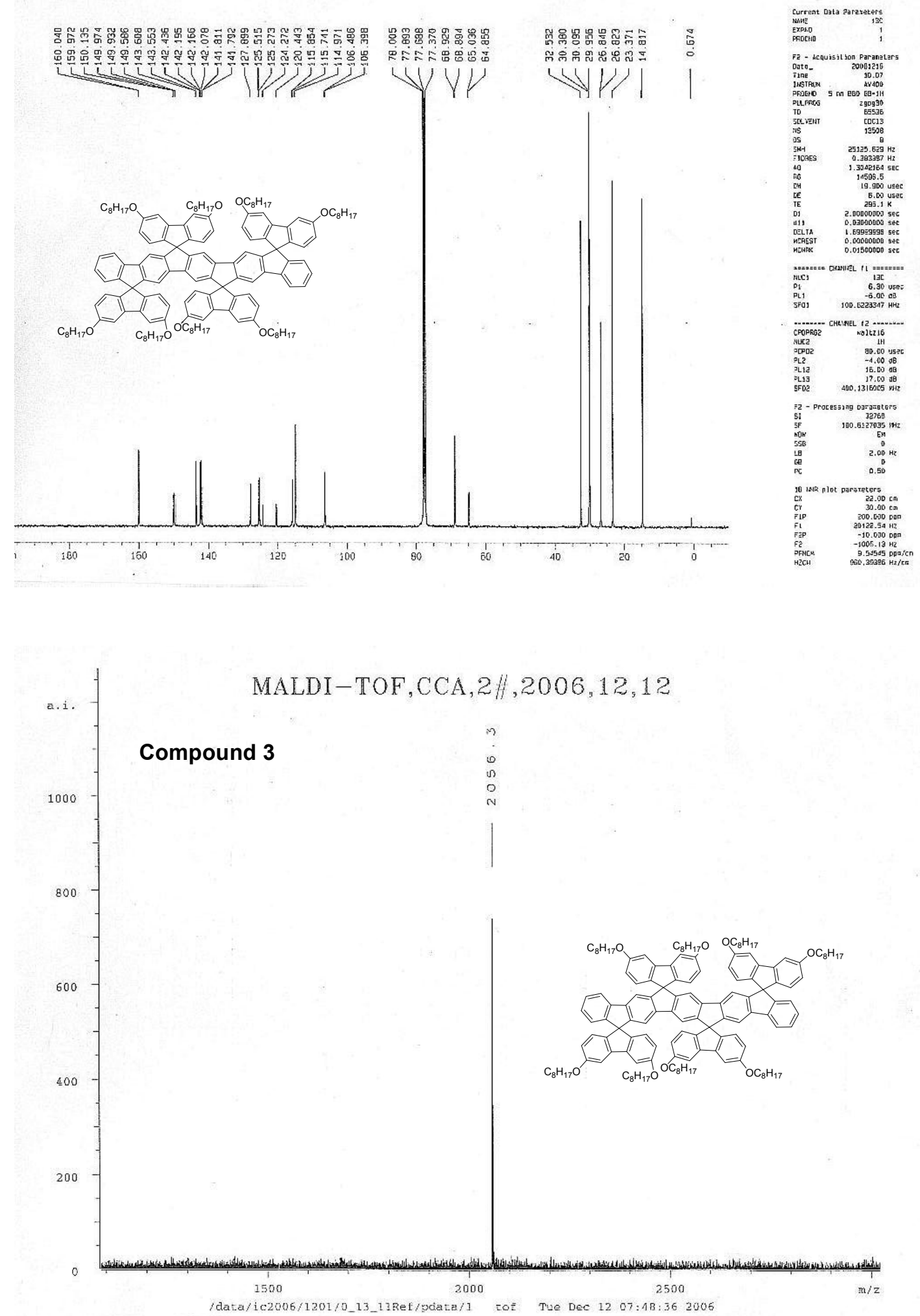

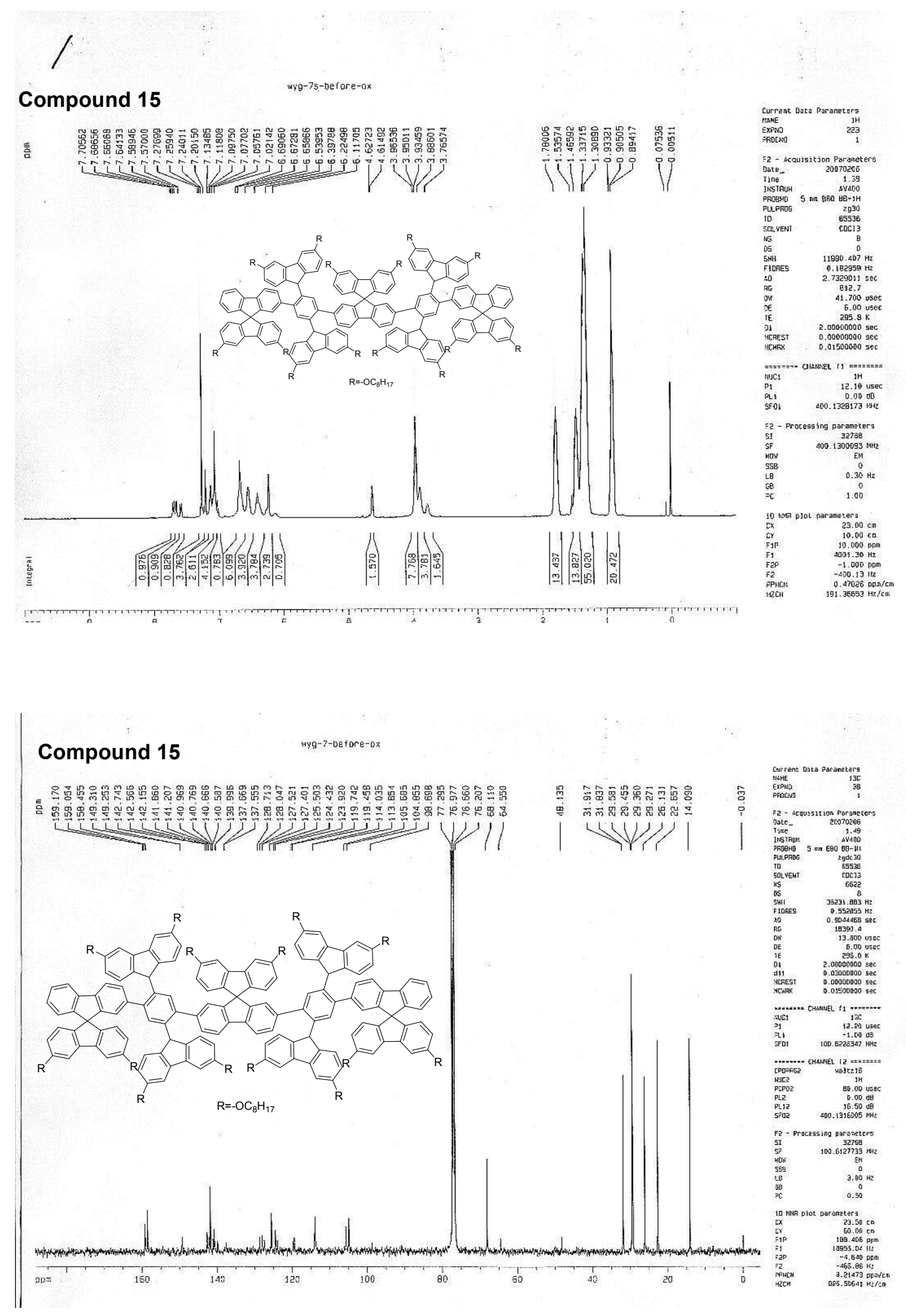


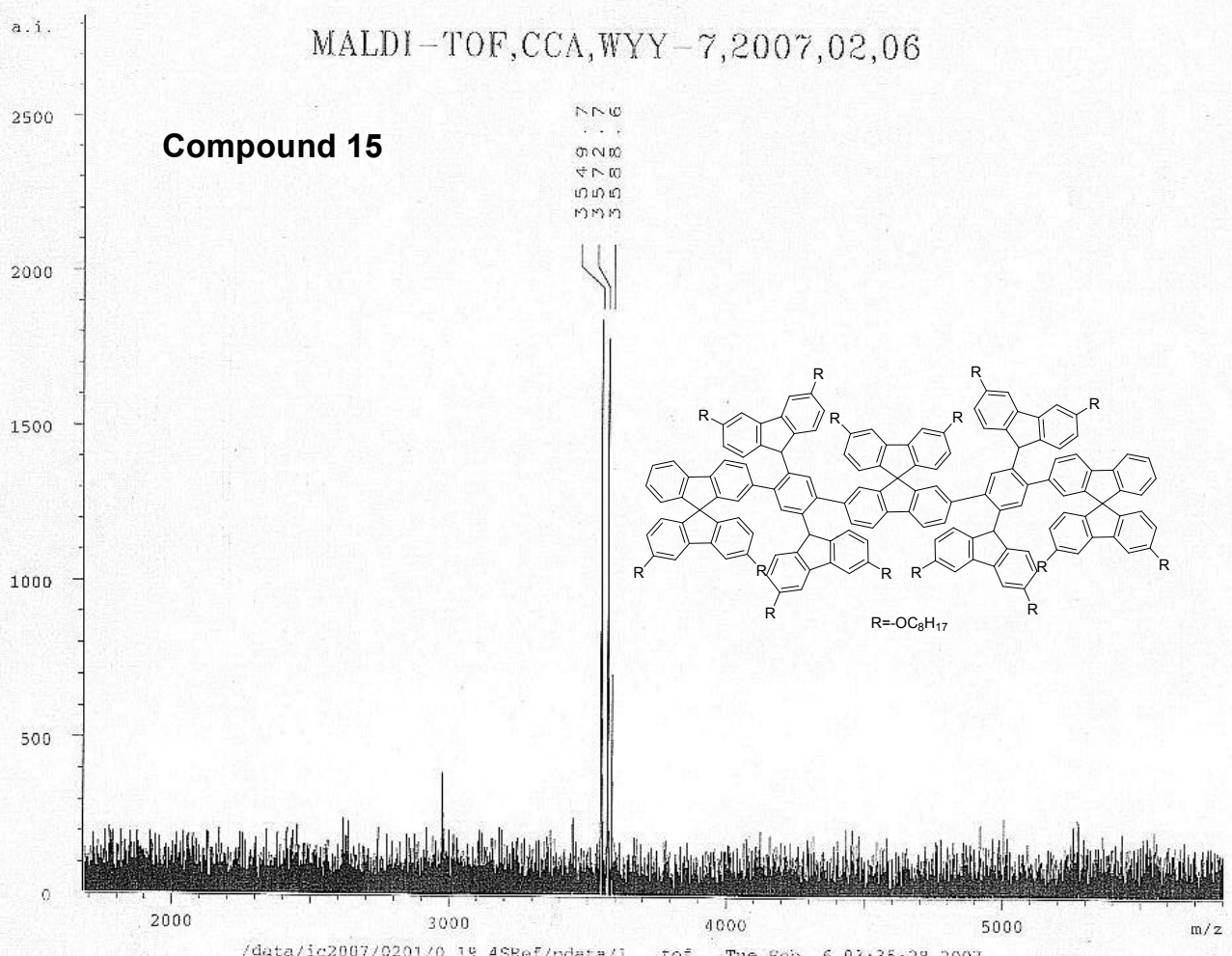

\section{Compound 4}
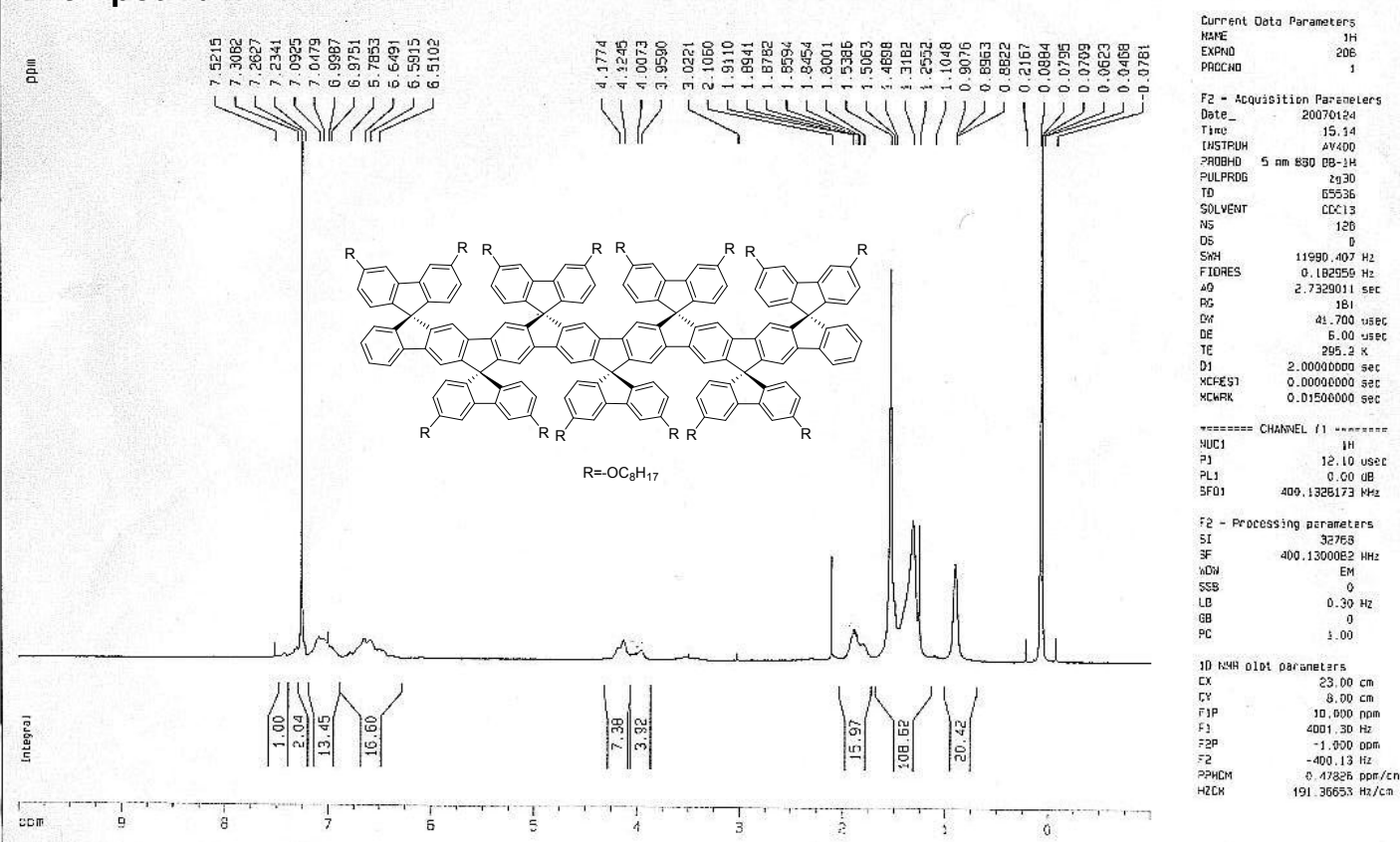


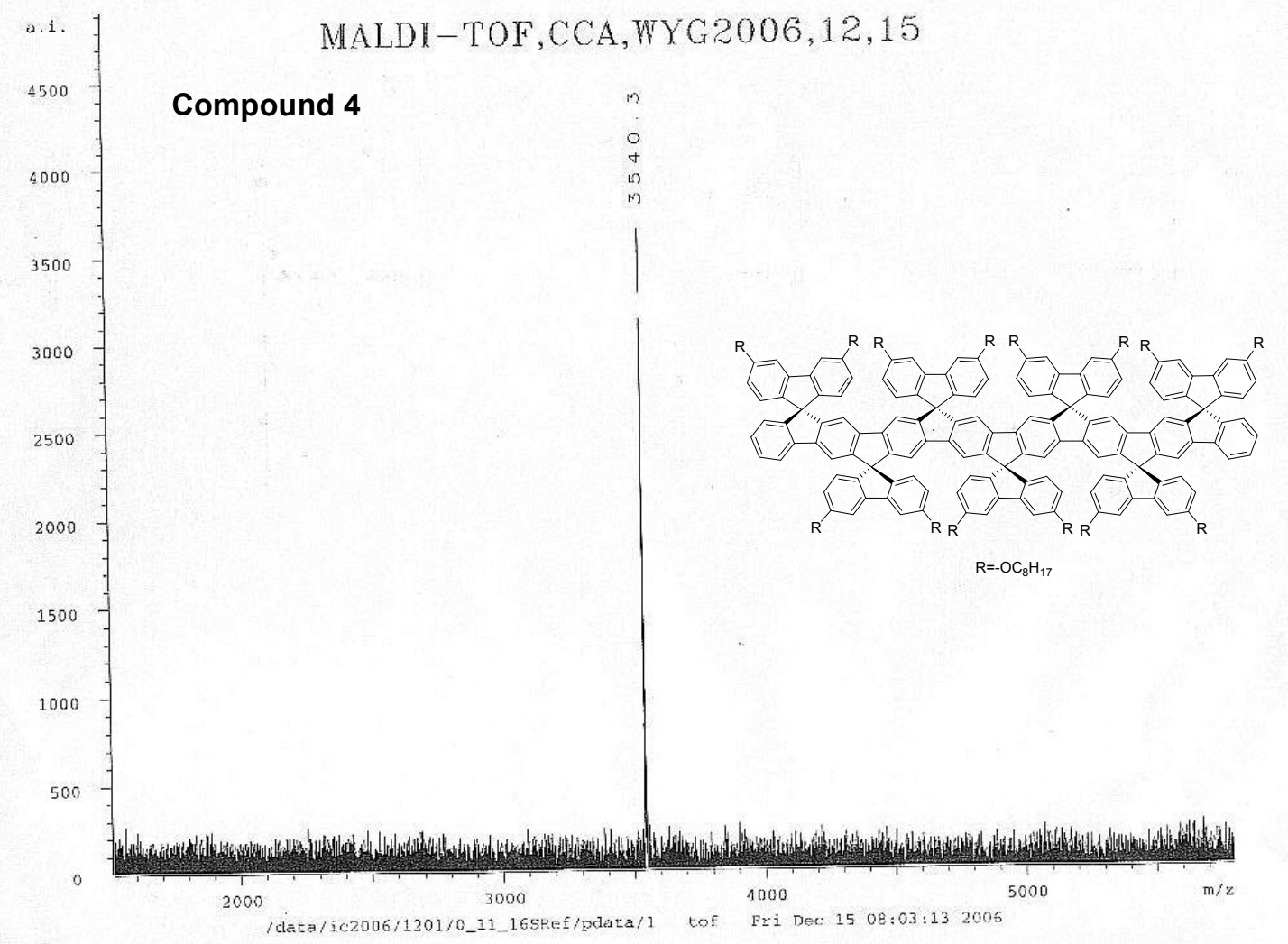

